\title{
Reciprocal Interareal Connections to Corticospinal Neurons in Mouse M1 and S2
}

\author{
(D)Benjamin A. Suter and Gordon M.G. Shepherd \\ Department of Physiology, Feinberg School of Medicine, Northwestern University, Chicago, Illinois 60611
}

Primary motor (M1) and secondary somatosensory (S2) cortices, although anatomically and functionally distinct, share an intriguing cellular component: corticospinal neurons (CSP) in layer 5B. Here, we investigated the long-range circuits of CSPs in mouse forelimb-M1 and S2. We found that interareal projections (S2 $\rightarrow \mathrm{M} 1$ and $\mathrm{M} 1 \rightarrow \mathrm{S} 2$ ) monosynaptically excited pyramidal neurons across multiple layers, including CSPs. Area-specific differences were observed in the relative strengths of inputs to subsets of CSPs and other cell types, but the general patterns were similar. Furthermore, subcellular mapping of the dendritic distributions of these corticocortical excitatory synapses onto CSPs in both areas also showed similar patterns. Because layer 5B is particularly thick in M1, but not S2, we studied M1-CSPs at different cortical depths, quantifying their dendritic morphology and mapping inputs from additional cortical (M2, contralateral M1, and local layer 2/3) and thalamic (VL nucleus) sources. These results indicated that CSPs exhibit area-specific modifications on an otherwise conserved synaptic organization, and that different afferents innervate M1-CSP dendritic domains in a source-specific manner. In the cervical spinal cord, CSP axons from S2 and M1 partly converged on middle layers, but S2-CSP axons extended further dorsally, and M1-CSP axons ventrally. Thus, our findings identify many shared features in the circuits of M1 and S2 and show that these areas communicate via mutual projections that give each area monosynaptic access to the other area's CSPs. These interareally yoked CSP circuits may enable M1 and S2 to operate in a coordinated yet differentiated manner in the service of sensorimotor integration.

Key words: corticocortical; corticospinal; motor; somatosensory; subcellular

\section{Introduction}

Primary motor cortex (M1) and secondary somatosensory cortex (S2), located on either side of primary somatosensory cortex (S1), are key areas in the cortical sensorimotor network. Their functions are diverse and complex, with M1 generally involved in movement-related functions and S2 in higher-order somatosensory processing. Recent studies in rodents have shown that separate classes of pyramidal neurons in S1 with distinct functional properties send different output signals to M1 and S2 (Chakrabarti and Alloway, 2006; Alloway, 2008; Sato and Svoboda, 2010; Chen et al., 2013; Yamashita et al., 2013). Indeed, M1 and S2 are proposed as major nodes in the mouse's dorsal "where" and ventral "what" pathways for somatosensation, respectively (Yamashita et al., 2013).

In contrast to these differences, M1 and S2 share an intriguing cellular constituent: corticospinal neurons (CSPs) (Burton, 1986; Li et al., 1990; Nudo and Masterton, 1990). Whereas M1-CSPs are extensively studied, S2-CSPs have received little attention de-

Received Oct. 16, 2014; revised Dec. 18, 2014; accepted Jan. 5, 2015.

Author contributions: B.A.S. and G.M.G.S. designed research; B.A.S. performed research; B.A.S. and G.M.G.S. analyzed data; B.A.S. and G.M.G.S. wrote the paper.

This work was supported by National Institutes of Health/National Institute of Neurological Disorders and Stroke Grants NS061963 and NS041234. We thank L. Trapp and D. Wokosin for technical assistance; N. Yamawaki for comments on the manuscript; L. Sybert for animal care; and T. Kiritani for helpful discussions.

The authors declare no competing financial interests.

Correspondence should be addressed to Dr. Gordon M. G. Shepherd, Northwestern University, Morton 5-660, 303 E. Chicago Avenue, Chicago, IL 60611. E-mail: g-shepherd@northwestern.edu.

DOI:10.1523/JNEUROSCI.4287-14.2015

Copyright $\odot 2015$ the authors $\quad 0270-6474 / 15 / 352959-16 \$ 15.00 / 0$ spite being a conserved feature of mammalian neocortex (Nudo and Masterton, 1990). CSPs in M1 and S2 share characteristic electrophysiological properties (Suter et al., 2013). The laminar organization of local excitatory circuits in S2 displays both similarities and differences compared with vibrissal M1 and S1 (Hooks et al., 2011). The synaptic circuits of S2-CSPs have not yet been directly investigated. A question of general interest for understanding cortical circuit organization is whether the long-range circuits of M1-CSPs and S2-CSPs have similar or specialized, area-specific features. A question of particular interest for understanding cortical mechanisms of sensorimotor integration is whether interareal M1 $\leftrightarrow$ S2 connections enable cross talk at the level of CSPs in each area.

Retrograde labeling studies have identified interareal projections between M1 and S2 in both directions in rodents (Donoghue and Parham, 1983; Burton, 1986; Carvell and Simons, 1987; Smith and Alloway, 2013). Electrophysiological dissection of the excitatory connectivity within these M1 $\leftrightarrow$ S2 pathways has not yet been undertaken but has the potential to illuminate the cellular specificity at the level of single cells and their dendritic arbors across different layers, and also how these areas fit into the corticocortical hierarchy. One intriguing possibility is that, whereas M2 $\rightarrow$ M1 (Hira et al., 2013; Hooks et al., 2013; Ueta et al., 2013, 2014) and S1 $\rightarrow$ M1 (Mao et al., 2011) projections follow feedback ("top-down") and feedforward ("bottom-up") patterns, respectively, the bidirectional connections between M1 and S2 may be reciprocally organized, implying a shared hierarchical level. 
We explored these issues by adapting photostimulation-based tools to characterize the reciprocal interareal excitatory inputs to CSPs in mouse M1 and S2. We focused on the lateral agranular region of $\mathrm{M} 1$ containing the forelimb representation area (Weiler et al., 2008; Tennant et al., 2011), and the corresponding forelimb area within S2 (see Materials and Methods). We quantified the topographies and amplitudes of monosynaptic connectivity patterns at the cellular and subcellular (dendritic) levels. The similarity in synaptic organization and the strong interareal coupling between M1 and S2 are greater than previously appreciated, suggesting parallel and coordinated corticospinal output across this sensorimotor cortical network.

\section{Materials and Methods}

Stereotaxic injections. All animal studies followed the guidelines of the National Institutes of Health and Society for Neuroscience for animal welfare and were approved by Northwestern University's Animal Care and Use Committee. Wild-type C57BL/6 mice of either gender were obtained from The Jackson Laboratory or bred in-house. At 5-6 weeks of age, mice were anesthetized with isoflurane in oxygen $(2.5 \%$ for induction, 1.5-2\% during surgery, Surgivet Isotec 4) and placed into a stereotax (David Kopf Instruments, Stoelting). Body temperature was maintained at $37^{\circ} \mathrm{C}$ by a feedback-controlled heating pad (FHC) inclined at $30^{\circ}$ to improve dorsal access to the cervical spinal cord. The head and/or upper back were shaved and disinfected and analgesic (buprenorphine, $0.2 \mathrm{mg} / \mathrm{kg}$ ) was administered subcutaneously. Surgical plane of anesthesia was confirmed and monitored every $20 \mathrm{~min}$ by toe pinch.

For cortical and thalamic injections, the scalp was infiltrated with lidocaine (2\%), a midline incision was made to expose the skull, and the origin of the stereotax was aligned to bregma. One or two small craniotomies ( $\sim 0.7 \mathrm{~mm}$ diameter; see below for injection coordinates) were made over the right hemisphere with a dentral drill (Osada, EXL-M40), the dura was removed, and the injection pipette was advanced into the brain under stereotactic control. Pipettes were pulled (Narishige PP-830) from borosilicate glass capillaries (Wiretrol II, Drummond Scientific) to an inner tip diameter of $35 \mu \mathrm{m}$, beveled (Narishige EG-400 Microgrinder), and tip-filled with retrograde tracer, virus (see below), or a mixture of virus and retrograde tracer. Tracers used included fluorescent microspheres (red or green Retrobeads IX, Lumafluor), used undiluted, and cholera toxin subunit B conjugated to Alexa-647, Alexa-594, or Alexa-488 (Invitrogen), used after dilution to $1 \mathrm{mg} / \mathrm{ml}$ in PBS (SigmaAldrich). At each injection site, 50-100 nl of solution was pressureinjected (40 psi, $10 \mathrm{~ms}$; Picospritzer III, Parker Instrumentation), and the pipette was left in place for 1-2 min before slowly retracting it.

For injections into the cervical spinal cord, a dorsal skin incision was made extending $\sim 1 \mathrm{~cm}$ caudal to lambda. Lidocaine was administered before and immediately after the incision. Custom-made retractors were inserted in the shoulder musculature to gain access to the spinal column, and longitudinal traction was applied via the retractors to increase separation between vertebrae. This approach permitted adequate access to the dorsal spinal cord between the $\mathrm{C} 2$ and $\mathrm{C} 3$ vertebrae, without requiring dissection of muscle or connective tissue. Beveled pipettes were prepared as above and inserted through the dura. Retrograde tracer $(\sim 1 \mu \mathrm{l})$ was pressure injected $50 \mu \mathrm{m}$ to the left of the midline, distributed between 0.4 and $1.0 \mathrm{~mm}$ below the dorsal cord surface. The pipette remained in place for $5 \mathrm{~min}$ at a depth of $0.4 \mathrm{~mm}$ before being slowly removed; the retractor was then gently removed.

Following injections, the incision was closed with sutures (plain gut, 1241B, Look) and adhesive (Super Glue, Loctite). The animal recovered on a heating pad, typically becoming ambulatory within 5 min after surgery and resuming normal behavior within $20 \mathrm{~min}$.

Injection coordinates. Injection pipettes were inserted vertically (relative to the flat skull surface around bregma), and coordinates were measured stereotactically relative to bregma; depths are relative to the pial surface. Coordinates for the secondary somatosensory cortex (S2) were as follows: $0.9 \mathrm{~mm}$ posterior, $4.0 \mathrm{~mm}$ lateral, $1.3 \mathrm{~mm}$ deep. Coordinates for the forelimb representation area of primary motor cortex (M1) were as follows: 0.6 and $1.2 \mathrm{~mm}$ anterior, $1.5 \mathrm{~mm}$ lateral, 0.8 and $0.3 \mathrm{~mm}$ deep.
Coordinates for the secondary motor area (M2) in the anterior frontal cortex were as follows: $2.7 \mathrm{~mm}$ anterior, $1.2 \mathrm{~mm}$ lateral, 0.7 and $0.3 \mathrm{~mm}$ deep. Coordinates for the ventrolateral (VL) nucleus of thalamus were as follows: 0.6 and $1.2 \mathrm{~mm}$ posterior, $1.1 \mathrm{~mm}$ lateral, $3.4 \mathrm{~mm}$ deep. Injection coordinates were developed based on retrograde tracing from forelimb M1, related studies in vibrissal M1, and standard anatomical atlases (Paxinos and Franklin, 2001; Hooks et al., 2011, 2013; Hunnicutt et al., 2014).

Viruses. Adeno-associated virus (AAV) stocks were obtained from the Penn Vector Core (University of Pennsylvania), stored at $-80^{\circ} \mathrm{C}$ in $10 \mu \mathrm{l}$ aliquots, thawed as needed, and then stored at $4^{\circ} \mathrm{C}$ for up to $4 \mathrm{~d}$ before use. To transfect neurons with channelrhodopsin-2 (ChR2), we used AAV2/1.CAG.ChR2-Venus.WPRE.SV40 (AV-1-20071P, Addgene 20071). For anterograde tracing experiments, we injected either AAV2/ 1.CAG.tdTomato.WPRE.bGH(AV-1-PV2126)orAAV2/1.CB7.CI.eGFP. WPRE.rBG (AV-1-PV1963).

In utero electroporation. We transfected cortical progenitor cells of the right motor cortex at embryonic day 16 by in utero electroporation of plasmids encoding ChR2, as previously described (Wood et al., 2009; Hooks et al., 2013). By postnatal week 4, a subset of layer $2 / 3$ neurons expressed ChR2 and Venus fluorescent protein. For these experiments, timed-pregnant females were obtained from Charles River Laboratories. Brain-slice experiments were performed on electroporated offspring at $\sim 4$ weeks of age.

Localization of M1 and S2. To target the forelimb representation area of M1 for injections and whole-cell recording, we relied on stereotaxic coordinates (see above) derived from atlases and prior intracortical microstimulation studies (Li and Waters, 1991; Weiler et al., 2008; Tennant et al., 2011). The band of retrogradely labeled CSP neurons reached its maximal thickness in this region of M1 (see Fig. 7B) (Anderson et al., 2010). Because S2 in the mouse lacks distinct cytoarchitectonic boundaries (Carvell and Simons, 1986), and as the surrounding areas do not project to the spinal cord (Burton, 1986), we localized S2 on the basis of labeled CSP neurons. Retrograde injections into the cervical spinal cord labeled a narrow band of CSP neurons within ventrolateral parietal cortex, dorsal to the rhinal sulcus (Nudo and Masterton, 1990). Within S2, we recorded from neurons in the forelimb representation area identified by anterograde innervation from forelimb M1 (see Fig. $7 F$ ). The localization of forelimb S2 is further strengthened by the presence of CSP neurons because $\mathrm{S} 2$ does not project to the lumbar spinal cord (see Results) (Burton, 1986). Injection coordinates for S2 (see above) were selected to target the same area where S2 whole-cell recordings were performed. Accurate placement of viral injections into S2 was routinely verified by inspecting the $\mathrm{S} 2$-containing brain slices and confirming that the injection site was located within the zone of labeled S2-CSPs.

Brain and spinal cord anatomy. For anatomical studies, mice were deeply anesthetized (200 mg/kg ketamine and $20 \mathrm{mg} / \mathrm{kg}$ xylazine) and transcardially perfused with $15-20 \mathrm{ml}$ of chilled low-divalent ACSF (as for electrophysiology experiments, but lacking calcium and magnesium), followed by $30 \mathrm{ml}$ of PFA ( $4 \%$ in ACSF, Electron Microscopy Sciences). The brain was extracted and immersed in PFA for $\sim 24 \mathrm{~h}$ at $4^{\circ} \mathrm{C}$, rinsed 3-4 times in PBS, and stored for up to 2 weeks at $4^{\circ} \mathrm{C}$. Coronal slices (100 $\mu \mathrm{m}$ thick) were cut on a vibrating microtome (Microm HM $650 \mathrm{~V}$ ).

To extract the spinal cord, first a complete laminectomy was performed at all spinal levels, and the nerve roots were transected; the skull was removed, and the cord and brain were removed in one piece. To stabilize the tissue for cutting, it was embedded in an agarose gel $(2.5 \%$ in PBS, Agarose Type 1-B, Sigma-Aldrich). The spinal cord was manually blocked into sections $\sim 1 \mathrm{~cm}$ in length, and then microtome-cut as above into transverse sections of $100 \mu \mathrm{m}$ thickness. Brain and spinal cord sections were mounted on glass slides and imaged on a macroscope (Olympus, SZX16) equipped with a solid-state plasma light source (Thorlabs, HPLS245) and a cooled CCD camera (QImaging, Retiga 2000R, 12-bit). Sections corresponding to cervical levels C1, C3, C6, and C8 were selected for high-resolution imaging on a two-photon microscope. In one case, this analysis was extended rostrally to include the pyramidal decussation.

For display, image contrast and brightness were globally adjusted in Fiji (Schindelin et al., 2012) and ImageJ software (Schneider et al., 2012). 
In one case (see Fig. 1E), the red channel was enhanced by spectral unmixing (open-source ImageJ plugin provided by Joachim Walter) (Neher et al., 2009) to compensate for bleed-through from the yellow Venus signal.

To quantify the horizontal extent of retrogradely labeled CSP neurons in coronal slices of $\mathrm{M} 1$ and $\mathrm{S} 2$, we used ImageJ software to measure the straight-line distance between the lateral and medial boundaries of CSP fluorescence. For each animal, we first calculated the average width across slices in each region, and in the text we report the mean and SEM across animals.

Two-photon microscopy. Fixed brain and spinal cord slices were imaged on a custom-built two-photon microscope running commercial software (Prairie, version 4). To ensure that physical distance and focal distance along the optical axis remained identical, the index of refraction was matched between the immersion medium (Type FF, Cargille) and mounting medium (Dako), and the correction collar on the objective lens ( $25 \times$, NA 0.8 , Zeiss) was adjusted to account for the presence of a glass coverslip $(0.17 \mathrm{~mm}$, Warner). Imaging of thick cortical sections $(300 \mu \mathrm{m})$ containing filled CSP neurons for the purpose of dendritic reconstructions typically required 3-6 tiled $z$-stacks, each containing $\sim 350$ slices with a $z$-step size of $0.84 \mu \mathrm{m}$. The laser was tuned to $780 \mathrm{~nm}$ for optimal excitation of Alexa-488 or Alexa-568 fluorescence. Imaging of axons in thin $(100 \mu \mathrm{m})$ spinal sections typically required $25-50$ tiled $z$-stacks, each containing $\sim 90$ slices with a $z$-step size of $3.0 \mu \mathrm{m}$. The laser was tuned to $975 \mathrm{~nm}$ to achieve balanced excitation of eGFP and tdTomato fluorescence. Emitted light was collected in two channels: green (eGFP or Alexa-488) from 490 to $560 \mathrm{~nm}$, and red (tdTomato or Alexa-568) from 580 to $620 \mathrm{~nm}$.

Dendritic reconstructions. We followed previously published methods to reconstruct the dendritic arbor morphology of neurons filled with biocytin during electrophysiological recordings (Suter et al., 2013). Briefly, slices were fixed, stained, and mounted. To avoid fluorescence quenching, the slides were sealed first with an organic sealant (CoverGrip, Biotium) and, once dry, then by nail polish. Two-photon image stacks were acquired as detailed above, and dendritic structures were digitally reconstructed in three dimensions (Neurolucida). Reconstructions were converted to the SWC file format using custom software. Each reconstruction was aligned in a common reference frame: a rotation was applied in the $x-y$ plane such that the apical axis of the neuron was parallel to the $y$-axis; a translation was applied to move the soma to the origin of the $x$-axis, and to locate the pia at the $y$-axis origin. To quantify dendritic length density, we superimposed a two-dimensional grid over the reconstruction and calculated the total reconstructed dendritic length within the three-dimensional volume extending through the full slice thickness at each grid square (Shepherd and Svoboda, 2005). The height and orientation of the grid ( 30 rows, 14 columns, $50 \mu \mathrm{m}$ spacing) were identical to the grid used for subcellular mapping (see below); it was $100 \mu \mathrm{m}$ wider on each side. Where individual sections (i.e., unbranched, tapered cylinders) of the reconstruction occupied multiple grid voxels, such sections were parametrically divided so that they contributed the appropriate length to each voxel. For analysis of how dendritic length density depends on soma depth, individual two-dimensional maps were converted to vertical profiles by summing the dendritic length across each row. To obtain a soma-aligned view of dendritic length density, this analysis was repeated with a modified coordinate system and grid: the soma was at the $x-y$ origin, and the grid had one row centered on the soma, 18 rows above the soma, and 6 rows below the soma. The dendritic length per compartment was measured by summing across the top 3 rows of pia-aligned profiles for the tuft, and the perisomatic region was defined as rows 14 and greater in the soma-aligned profiles. These reconstructions will be submitted to www.NeuroMorpho.Org.

Slice preparation. Several (2-3) weeks after virus injection, adult mice (age $8-9$ weeks) were deeply anesthetized with isoflurane and rapidly decapitated. A blocking cut was made caudal to the region of interest (M1 or S2). For recordings in $\mathrm{M} 1$, the blade was tilted anteriorly $15^{\circ}$ from the coronal plane. For recordings in S2 of the right hemisphere, the blade was first tilted anteriorly $15^{\circ}$ from coronal, and then rotated $20^{\circ}$ counterclockwise. The tissue block was then mounted in a rostral-up orientation, and brain slices were prepared as previously described (Suter et al., 2013).
Electrophysiology. Whole-cell recordings in brain slices were performed as previously described (Suter et al., 2013). Slices were bathed in ACSF (in mM: $127 \mathrm{NaCl}, 25 \mathrm{NaHCO}_{3}, 25$ D-glucose, $2.5 \mathrm{KCl}, 1 \mathrm{MgCl}_{2}, 2$ $\mathrm{CaCl}_{2}, 1.25 \mathrm{NaH}_{2} \mathrm{PO}_{3}$ ) at $23^{\circ} \mathrm{C}$. Epifluorescence microscopy was performed using custom-built LED illumination (M470L2, M530L2, M660L3, Thorlabs) and standard filter sets (U-N31002, U-N41017, Chroma; Cy5-4040C, Semrock). Recordings were targeted to regions of cortex where anterogradely labeled axons overlapped with retrogradely labeled CSP neurons. Individual neurons of interest were identified at high magnification (LUMPlanFL N 60×, NA 1.00, Olympus) based on their laminar location, pyramidal soma shape, retrograde label, and depth below slice surface $(50-100 \mu \mathrm{m})$. In experiments using multiple retrograde tracers, after identifying a neuron of the desired projection type, we confirmed the absence of the other retrograde tracer type. For whole-cell recordings, borosilicate pipettes (BF150-86-7.5, Sutter) were filled with a potassium-based internal solution (in mM: $128 \mathrm{KMeSO}_{4}, 10$ HEPES, $1 \mathrm{~K}_{2}$-EGTA, $4 \mathrm{MgCl}_{2}, 4 \mathrm{Na}_{2}$-ATP, $0.4 \mathrm{Na}$-GTP, $10 \mathrm{Na}_{2}$ phosphocreatine, 3 ascorbate, $\mathrm{pH} 7.3)$ containing biocytin $(2-4 \mathrm{mg} / \mathrm{ml}$, Sigma-Aldrich) and (in most experiments) also a fluorescent dye (Alexa$647,50 \mu \mathrm{M}$, Invitrogen) to visualize the neuron's dendritic morphology after completion of the recordings and confirm that the slice angle was appropriate to maintain intact apical arbors. Once a gigaohm seal was obtained, the high-magnification immersion lens was removed from the bath and the slice surface was brought into focus at low magnification, in preparation for optical stimulation. After breaking in, we waited 3 min before commencing stimulus trials. Traces were acquired in voltage-clamp mode at a command potential of $-70 \mathrm{mV}$. Different photostimulation paradigms were used, as described in detail below. For all types of electrophysiological experiments, data acquisition and synchronized control of experimental apparatus were performed with open-source Ephus software (www.ephus.org) (Suter et al., 2010).

Subcellular ChR2-assisted circuit mapping (sCRACM) conditions. We used pharmacological conditions, developed for sCRACM (Petreanu et al., 2009), that isolate monosynaptic responses evoked by photostimulation. We added a mixture of drugs to the bath that collectively abolishes action potentials and hence polysynaptic transmission (TTX, $1 \mu \mathrm{M}$, Tocris Bioscience), while at the same time enhancing depolarization of ChR2-positive axon terminals sufficiently to induce synaptic transmission (4-AP, $100 \mu \mathrm{M}$, Sigma-Aldrich). We also blocked NMDAR-mediated transmission (CPP, $5 \mu \mathrm{M}$, Tocris Bioscience) to avoid nonlinear dendritic responses. The sCRACM conditions were used for both subcellular mapping based on laser scanning and wide-field photostimulation, as indicated in Results and further explained in the Materials and Methods sections that follow.

Wide-field photostimulation. After obtaining a gigaohm seal at high magnification, we switched to a low-magnification air objective (UPlanSApo $4 \times$, NA 0.16, Olympus). When comparing input with sequentially recorded pairs of neurons, recordings were targeted to neurons close to each other (within $\sim 50 \mu \mathrm{m}$ ). When recording multiple neurons at varying cortical depths (laminar or sublaminar profiles), the lateral separation was $<100 \mu \mathrm{m}$ and the stage was translated to the same vertical position for each neuron in the group to ensure equivalent synaptic stimulation over the entire cortical depth. Horizontal profiles were recorded from neurons at similar cortical depths; the stage was initially centered on the first neuron and translated horizontally to center each subsequent neuron's dendritic arbor. To avoid potential confounds due to stimulation sequence, the cell-type order was alternated when recording labeled pairs and the laminar order was randomized when recording profiles. Blue light from an LED (M470L2, driver: LEDD1B, Thorlabs) passed through the epifluorescence light path of the microscope, including an excitation filter (HQ480/20×, Chroma), and produced even illumination over an area greater than the region of interest ( $\mathrm{M} 1$ or S2). The LED was controlled by the data acquisition software, and stimulation intensity was measured with a calibrated power meter (PM100D, S130C, Thorlabs). In each trial, a 5 ms stimulus was preceded by a $100 \mathrm{~ms}$ baseline period. Stimulation intensity was $1 \mathrm{~mW} / \mathrm{mm}^{2}$. Stimuli were repeated 3-4 times with $45 \mathrm{~s}$ intervals while recording somatic current responses in voltage-clamp mode. 
Laser-scanning photostimulation. sCRACM was performed as previously described (Petreanu et al., 2009) on a custom-built laser-scanning microscope (laser: 473 nm, 50 mW, MLL-FN473, CNI Laser; scanner: model 6210 mirror galvanometer pair, Cambridge Technologies; shutter: LS2ZM2, VCM-D1, Uniblitz; Pockels cell: 350-50, 302 RM, Conoptics). After establishing a whole-cell recording and centering the cortex in the video image, a graphical user interface for mapping was used to mark the location of the soma (as inferred from the pipette), and a grid of stimulation sites was overlaid onto the bright-field image. The stimulation grid consisted of 30 rows and 10 columns, with uniform $50 \mu \mathrm{m}$ spacing between sites. The upper edge of the grid was aligned to the pia, and the grid was then centered on the soma. The height of the grid $(1500 \mu \mathrm{m})$ was chosen to ensure coverage across all layers and into white matter. The width of the grid $(500 \mu \mathrm{m})$ was chosen based on the maximal dendritic dimensions observed in our reconstructions. At the beginning of each experiment, laser power was adjusted (between 0.1 and $2.0 \mathrm{~mW}$ at the back focal plane) to elicit $\sim 100$ pA peak responses for stimulation near the soma (Petreanu et al., 2009). Each neuron was mapped 2-3 times, and in each repetition the 300 stimulation sites were visited in a different pseudorandom sequence. In a subset of experiments, the slice was rotated by $180^{\circ}$ before patching, to detect potential orientation-dependent spatial errors; maps from rotated slices appeared similar and were thus pooled with the upright maps.

Data analysis. Quantification and analysis for electrophysiology data were performed with custom routines in MATLAB (MathWorks). All responses were recorded in voltage-clamp mode, and inward currents are reported as positive values. No offline filtering was applied. To quantify responses to wide-field photostimulation, the baseline was subtracted (50 ms prestimulus), and repeated traces were averaged. The resulting average current response was summed over the $100 \mathrm{~ms}$ following stimulus onset, and converted to units of charge $(\mathrm{pC})$. All responses returned to baseline within $100 \mathrm{~ms}$ after stimulus. Because viral expression of ChR2 is variable, we recorded from multiple neurons (pairs or profiles) within each slice. Comparisons between neurons recorded within the same slice are displayed in units of charge. For comparison across multiple slices, response amplitudes were normalized to the mean amplitude within the slice. For laminar profiles, each neuron's cortical depth relative to the pia was measured post hoc in bright-field images, and converted to a normalized depth (fraction of the cortical thickness). The same procedure was used for sublaminar profiles and the normalized pooled data were fit by linear regression. For horizontal profiles, we measured the horizontal distance from a common landmark to each neuron recorded within a slice. Input amplitude was then plotted against horizontal position, and the resulting profile was centered. Profiles from multiple slices were amplitude-normalized to perform a Gaussian fit to the pooled data points. To quantify responses to laser-scanning photostimulation maps, the response at each stimulation site was analyzed as follows: the baseline (100 ms prestimulus) was subtracted and the resulting response averaged over the $80 \mathrm{~ms}$ following stimulus onset. Traces where the baseline period showed large fluctuations ( $>5$ SDs above the baseline variability calculated across the entire map) were discarded. Finally, we averaged across repeated maps. Individual input maps are displayed as a two-dimensional matrix, where the color at each pixel encodes the response at the corresponding stimulation site. We normalized each map to its maximal pixel value (Petreanu et al., 2009). For some analyses, we collapsed each map to a vertical profile by summing across each row, and normalized to the maximum pixel value.

Details of statistical testing are provided in Results. Comparisons used a Wilcoxon rank-sum test, or a Wilcoxon signed-rank test for paired datasets. Significance was defined as $p<0.05$. When making multiple comparisons, this value was Bonferroni-corrected. Linear regressions were considered significant when the $95 \%$ confidence interval did not include zero (and $p$ values are reported for an $F$ test on the regression relationship).

\section{Results}

\section{S2 axons monosynaptically excite M1 neurons across all layers}

This study examined long-range circuits of CSPs located both in the forelimb representation region (lateral agranular cortex; area
4) of primary motor cortex (M1) and in the secondary somatosensory cortex (S2), with a focus on the mutual projections of the two areas (i.e., the interareal corticocortical pathways in both directions) and how they innervate CSPs. For clarity, we first present the set of results concerning S2 inputs to M1-CSPs, followed by those for M1 inputs to S2-CSPs.

To assess $\mathrm{S} 2 \rightarrow \mathrm{M} 1$ projections, we first localized the upstream source of these in S2 by injecting retrograde tracer into M1 and thereby labeling M1-projecting neurons in S2. In the same mice, as a second and complementary way to localize S2 based on the presence of CSPs, we also injected retrograde tracer of a different color in the spinal cord, thereby labeling S2-CSPs (Fig. 1A). We observed retrogradely labeled M1-projecting neurons in S2, which overlapped with the zone of S2-CSP labeling (Fig. 1B). These M1-projecting neurons were never double-labeled with the spinal tracer, and were located across multiple S2 layers except layers 1 and 4 (Fig. $1 B$ ). This result indicates that $\mathrm{S} 2$ contains numerous M1-projecting neurons.

Next, to anterogradely label the axons of M1-projecting S2 neurons and visualize their projection to M1, we injected virus encoding eGFP (AAV-eGFP) into S2 and injected retrograde tracer in the spinal cord (Fig. 1C). In subsequently prepared M1 slices, we observed fluorescently labeled S2 axons in M1 (Fig. 1D), with a banded appearance across layers. This pattern of S2 axon branching in M1 is also broadly consistent with that observed for $\mathrm{S} 2 \rightarrow \mathrm{M} 1$ projections in the rat (Smith and Alloway, 2013) in that the axons terminate in a relatively narrow zone in M1, but differs in that this zone appeared slightly more laterally situated in the mouse. Labeled S2 axons were also observed in more rostral and medial areas outside the forelimb M1 region and were not studied further here.

To assess the excitatory synaptic connections formed by S2 axons onto M1 neurons, including M1-CSPs, we injected virus encoding ChR2 (AAV-ChR2-Venus) into S2 and injected retrograde tracer in the spinal cord (Fig. $1 E$ ). Subsequently, we prepared M1 slices and again observed fluorescent S2 axons (Fig. $1 F)$. In these slices, we assayed $\mathrm{S} 2 \rightarrow \mathrm{M} 1$ connectivity by combining wide-field photostimulation of ChR2-expressing S2 axons with whole-cell recordings of evoked excitatory synaptic responses in M1 neurons. We added TTX and 4-AP to the bath solution to isolate monosynaptic inputs (see Materials and Methods). In each slice, we sampled from multiple neurons across layers to obtain a laminar profile (Fig. $1 F$ ). To control for sliceto-slice variability in expression levels, we normalized the responses in each laminar profile to the mean value and plotted these as a function of the normalized soma depth in the cortex $(0=$ pia; $1=$ white matter) (Fig. $1 G)$. S2 input was strongest in layer $2 / 3$ and decreased with cortical depth (Fig. $1 G, H$ ). This laminar pattern of $\mathrm{S} 2 \rightarrow \mathrm{M} 1$ innervation resembles the innervation of mouse vibrissal M1 (medial agranular area) neurons by axons from both S1 (Mao et al., 2011) and sensory thalamus (PO nucleus) (Hooks et al., 2013) in that layer $2 / 3$ received strong inputs, but differed in that here deeper-layer neurons also received substantial input.

\section{S2 axons similarly excite CSP, S2-projecting, and callosally projecting neurons in layer $5 \mathrm{~B}$ of $\mathrm{M1}$}

Next we investigated whether the S2 inputs to layer 5B neurons depended on the postsynaptic neuron's projection class, by recording from CSPs and comparing their responses to neighboring pyramidal neurons in layer $5 \mathrm{~B}$ identified as S2-projecting corticocortical neurons. For this, in addition to injecting S2 with AAV-ChR2, we also injected retrograde tracers (different colors) into the spinal cord and S2 (Fig. 2A). We then selected a pair of 
A

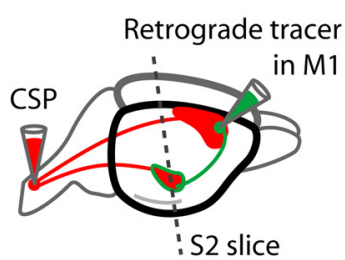

C

AAV-eGFP in $S 2$

E

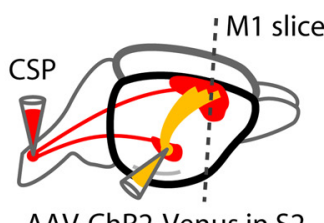

AAV-ChR2-Venus in S2

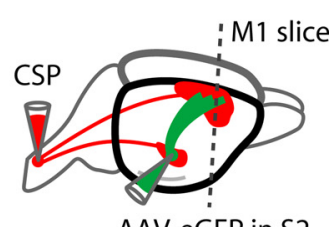

B

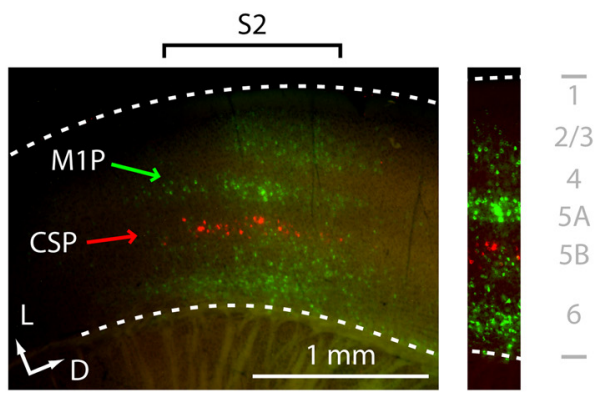

D

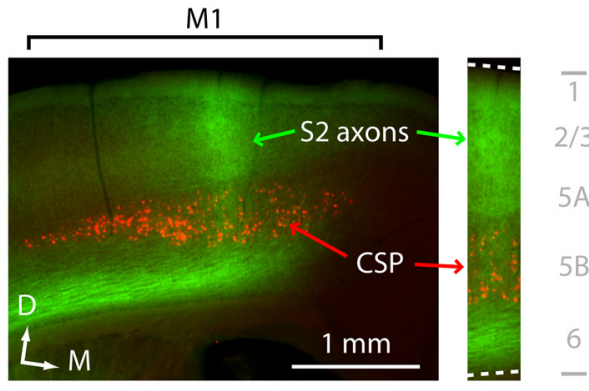

$\mathbf{F}$
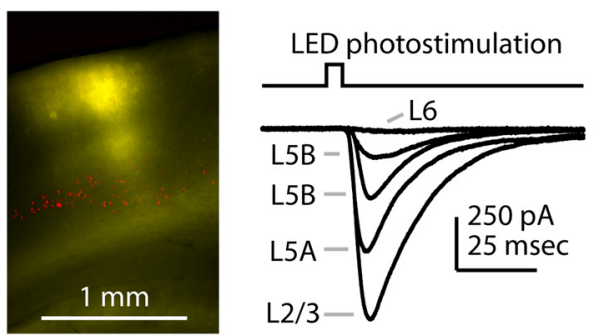

G

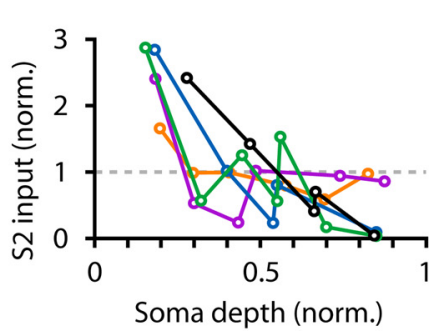

H

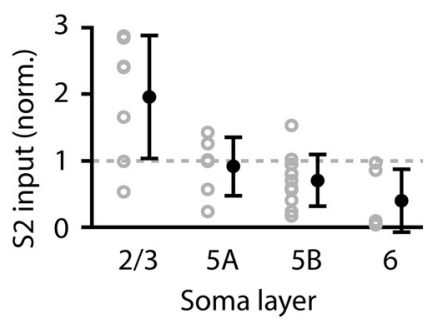

Figure 1. S2 axons monosynaptically excite M1 neurons across all layers. $\boldsymbol{A}$, Labeling paradigm. Retrograde tracer injections were made in the spinal cord and M1, and S2 slices were examined for labeling of CSP (red) and M1-projecting corticocortical (M1P) neurons (green). $\boldsymbol{B}$, Left, Epifluorescence image of labeling pattern in S2 slice. D, Dorsal; L, lateral. Right, Higher-magnification view of laminar distribution of retrogradely labeled neurons. WM, White matter. C, Labeling paradigm. Retrograde tracer was injected in the spinal cord, AAV-eGFP was injected in S2, and M1 slices were prepared to visualize S2 axons in M1. D, Left, Epifluorescence image, showing S2 axons (green) and CSPs (red) in M1. D, Dorsal; M, medial. Right, Higher-magnification image, showing S2 axons in all layers (green; arrows) overlapping with M1-CSPs (red; arrow). $\boldsymbol{E}$, Labeling paradigm. Retrograde tracer was injected in the spinal cord, AAV-ChR2-Venus was injected in S2, and M1 slices were prepared to visualize and photostimulate S2 axons while recording from CSP and other M1 neurons. $\boldsymbol{F}$, Left, Example epifluorescence image. Right, Example voltage-clamp traces of photo-evoked responses. Wide-field illumination with a gated LED was used to photostimulate the ChR2-expressing $S 2$ axons (yellow) while recording from M1 neurons in different layers (layer 2/3, etc., as indicated). G, Laminar profiles from several slices, plotted together after normalization (to the mean input per slice). Soma depth was normalized: $0=$ pia; $1=$ white matter The black profile corresponds to the example traces in $\boldsymbol{G}$. $\boldsymbol{H}$, Same data as in $\boldsymbol{G}$, but with the values pooled across profiles by layer (gray) and averaged (black) (mean \pm SEM).

neighboring pyramidal neurons of each type (CSP and S2projecting) for sequential recordings (Fig. $2 B$ ). No doublelabeled neurons were observed, confirming that these are nonoverlapping projection classes. Responses in CSP and S2projecting neurons in layer $5 \mathrm{~B}$ varied from pair to pair, but overall did not differ in amplitude ( $p=0.46$ ) (Fig. 2C). To confirm and extend these findings, we repeated this experiment but recorded from callosally projecting (CAL) neurons in layer $5 \mathrm{~B}$ of
M1, labeled by injecting contralateral M1 instead of ipsilateral S2. This, too, showed that CSP and CAL neurons in layer 5B of M1 were on average similarly innervated by axons from $\mathrm{S} 2(p=0.38)$ (Fig. $2 D-F)$. These results thus indicate that $\mathrm{S} 2$ axons similarly innervate layer $5 \mathrm{~B}$ neurons in M1, independent of projection class. Furthermore, S2 axons monosynaptically excite M1-CSPs, forming a basis for a long-range corticocortical $\rightarrow$ corticospinal pathway, and they also monosynaptically excite S2projecting neurons in M1, forming a basis for reciprocal coupling (i.e., a feedback loop) between the two areas.

\section{S2 axons excite M1-CSP over a narrow horizontal range, strongest in upper layer 5B}

As noted above, CSP labeling in M1 extended horizontally over a broad swath of cortex, but S2 axons were restricted to a columnar subregion of M1 (see also Smith and Alloway, 2013), suggesting that only a subset of CSPs receive excitatory input from S2 axons. To test this, we obtained horizontal profiles of S2 input by recording from CSPs at a range of horizontal distances from the center of the S2 axon column (Fig. $3 A-C$ ). The pooled data across animals $(n=3)$ showed that the zone of S2 innervation in M1 was on the order of $\sim 1 \mathrm{~mm}$ in width (Fig. 3D). In contrast, in these coronal slices, the labeled CSPs were horizontally distributed over $\sim 2 \mathrm{~mm}$ (mean $\pm \mathrm{SEM}, 1.99 \pm 0.08$ $\mathrm{mm}$ ). Thus, S2 axons innervated a subregion of the M1-CSP territory, similar to the projection from S2 to rat motor cortex as described anatomically (Smith and Alloway, 2013).

Labeled CSPs in M1 were distributed over the full width of layer $5 \mathrm{~B}(\sim 25 \%$ of total cortical thickness). Because neurons in upper and lower sublayers of layer 5B can be differentially targeted by axons from upstream sources (Yu et al., 2008; Anderson et al., 2010; Hooks et al., 2013), we therefore investigated whether S2 inputs similarly varied with CSP soma depth. We recorded from multiple CSPs located at different depths in the slice, and photo-excited S2 axons (Fig. 3E,F), generating a laminar profile for each slice spanning most of the thickness of layer $5 \mathrm{~B}$ (Fig. 3G). These results showed that S2 inputs to CSPs decreased substantially in amplitude with increasing soma depth in layer 5B (Fig. 3G,H). This gradient is similar to that observed for local inputs from layer $2 / 3$ (referred to above) but is the opposite of, and therefore complementary to, that observed for inputs to layer $5 B$ neurons from secondary motor cortex (M2, in the anterior frontal cortex), as observed in vibrissal M1 (Hooks et al., 2013). 
Dendritic morphology of M1-CSP is mostly independent of soma depth Could the differential innervation by S2 axons of CSPs across different depths in layer 5B simply reflect dendritic morphology differences? To assess this, we imaged dye-filled CSPs located at different depths in layer $5 \mathrm{~B}$, reconstructed their dendritic arbors (Fig. 4A), and analyzed these structures by converting them to maps of dendritic length density (Brecht and Sakmann, 2002; Shepherd et al., 2005) (Fig. 4B). CSPs located in the upper, middle, and lower part of layer $5 \mathrm{~B}$ had similar morphology, including numerous basal/perisomatic branches, an ascending apical dendrite with relatively few branches, and a high density of apical tuft branches mostly in layer 1. Comparing three such CSPs by overlaying their length density maps suggested that the main difference was simply in the depth (but not shape or size) of their basal/perisomatic arbor (Fig. $4 C)$. To explore this further, we reconstructed a larger sample of CSPs located across the full thickness of layer 5B (Fig. 4D). We analyzed their length density maps by converting each to a single vector, then pooled these vectors $(n=$ 24) and sorted them by soma depth, with the vectors aligned either to the pia or the soma (Fig. 4E). From these data, it appeared that the main difference among CSPs at different depths was the length of the mid-apical segment linking the perisomatic and apical-tuft regions, an impression borne out by quantitative analyses (linear regression of dendritic length vs soma depth) (Fig. $4 F$ ). Although total dendritic length (overall mean \pm SD: $1.27 \pm 0.12 \mathrm{~cm})$ increased slightly with depth $\left(R^{2}=0.19\right.$; slope $5.1 \mathrm{~mm} / \mathrm{mm}[0.4$ to $9.9,95 \% \mathrm{CI}] ; p=0.035)$, the only compartment to show a significant dependence on soma depth was the mid-apical segment $\left(R^{2}=0.60\right.$; slope $3.9 \mathrm{~mm} / \mathrm{mm}$ [2.5 to 5.3 , $\left.95 \% \mathrm{CI}] ; p=8 \times 10^{-6}\right)$. These results thus demonstrate a simple and continuously graded scaling relationship for the dendritic arbors of CSPs at different cortical depths. These findings are consistent with those for CSPs in rat M1, where morphology did not vary across electrophysiologically defined CSP subclasses (Tseng and Prince, 1993). They both resemble and contrast with findings in rat M2, which show depth-dependent dendritic differences, but with classification into discrete morphologically defined CSP subclasses (Ueta et al., 2014). Here, although depthdependent differences were pronounced enough to allow CSPs to be grouped into subclasses according to soma depth (Fig. 4C, G), differences between the groups were due to variation along a continuum.

\section{S2 axons innervate M1-CSP perisomatically and along apical dendrites except in layer 1}

Next, we investigated the subcellular organization of S2 inputs to M1-CSPs. For this, we used the sCRACM technique (see Materials and Methods), which enables mapping of the dendritic locations of monosynaptic inputs from ChR2-expressing presynaptic axons to a single postsynaptic neuron (Petreanu et al., 2009) (Fig. $5 A$ ). These maps provide a soma-centric view of dendritic input, where the effect of distal synapses is reduced as a result of electrotonic attenuation; however, the technique has sufficient sensitivity to detect strong inputs to distal dendrites (Petreanu et al.,
2009). Maps were peak-normalized for display, allowing comparison of the patterns but not amplitudes across cells. We injected the spinal cord with retrograde tracer, and S2 with AAV-ChR2Venus, yielding M1 slices with retrogradely labeled CSPs and ChR2-expressing S2 axons (Fig. 5B). In sCRACM maps recorded from CSPs located at different depths in layer $5 \mathrm{~B}$, the inputs from S2 axons occurred mostly at perisomatic sites, with some neurons also receiving strong apical dendritic inputs (Fig. $5 C$ ). This pattern was evident in the average map, which furthermore indicated a paucity of input to the distal apical tuft dendrites in layer 1 (Fig. 5D). This pattern was also generally consistent with the laminar pattern of the labeled S2 axons, which were distributed across multiple layers, with relatively high density in layer $2 / 3$ (Fig. 5B). To assess cell-to-cell differences as a function of soma depth, we also generated a "side-view" map of the grouped data by converting each neuron's map to a vector (by averaging along map rows), and sorting the collection of vectors by soma depth (Fig. 5E). This showed a generally consistent pattern across neurons of relatively strong perisomatic inputs plus variable amounts of apical dendritic input, but little or no distal apical input (top map rows). There was a tendency for the perisomatic inputs to occur slightly above (closer to the pia) the soma, more pronounced for higher CSPs (Fig. 5E). The lack of input to distal tufts in layer 1 was observed for CSPs at all depths, rather than just the deepest CSPs where electrotonic attenuation of distal synaptic input would be greatest. Additional analyses will be presented below, in comparison to other input pathways.

\section{Axons from four other sources innervate M1-CSPs in source-} specific subcellular input patterns

The dendritic topography of S2 inputs to M1-CSPs raises the question how this particular pattern compares with other inputs. We therefore performed additional sCRACM experiments to examine multiple other sources of input to M1-CSPs.

First, we examined input from thalamus, the main subcortical source of excitation to M1, focusing on the VL nucleus, shown to monosynaptically innervate layer $5 \mathrm{~B}$ pyramidal neurons in vibrissal M1 (Hooks et al., 2013). For this, we injected the VL nucleus in the thalamus with AAV-ChR2-Venus and spinal 
A

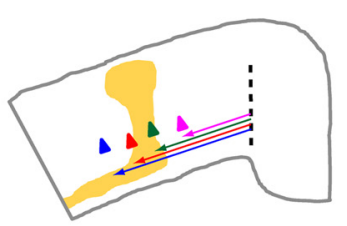

B

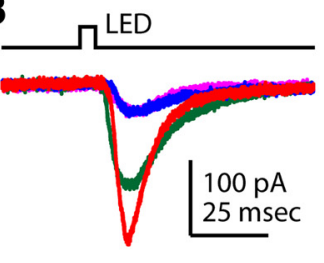

E
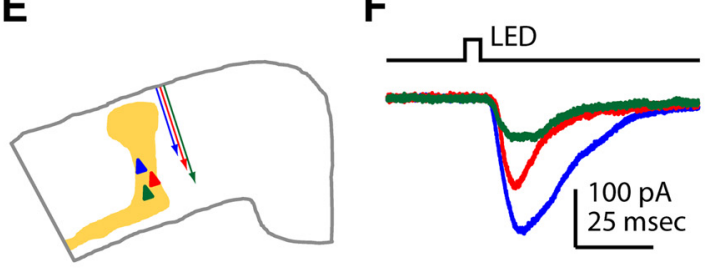

C

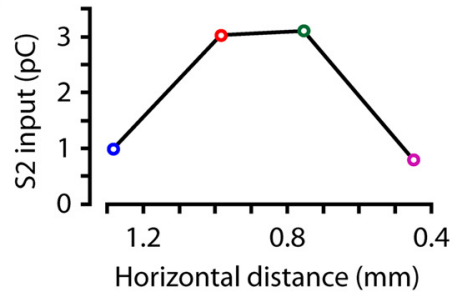

G

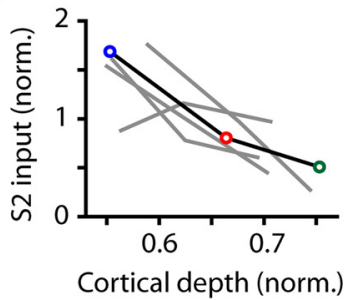

D

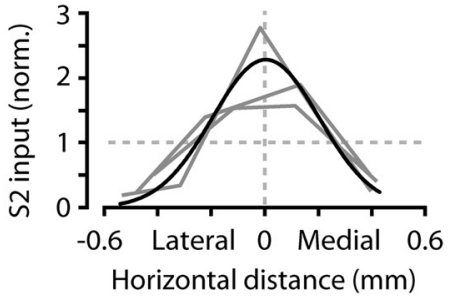

H

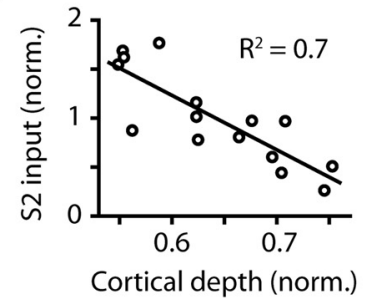

Cortical depth (norm.)

Figure 3. S2 axons excite M1-CSP over a narrow horizontal range, strongest in upper layer 5B. $A$, Recording paradigm. M1 slice indicating four horizontal positions for CSP recordings, and labeled $S 2$ axons. $\boldsymbol{B}$, Example traces recorded from CSPs at different horizontal positions across the $S 2$ axon zone; colors represent relative positions in $\boldsymbol{A}$. $\boldsymbol{C}$, Example horizontal profile corresponding to the traces in $\boldsymbol{B}$. Horizontal distance is relative to a medial landmark. $\boldsymbol{D}$, Profiles from three animals. Profiles were amplitude-normalized to the average value per profile and horizontally centered (equal area under the curve on each side of zero). Black curve represents Gaussian fit to the pooled data points after normalization and alignment. $\boldsymbol{E}$, Recording paradigm. M1 slice indicating (SP recordings made over a range of soma depths. $\boldsymbol{F}$, Example traces recorded within a single slice; colors represent relative depths in $\boldsymbol{E}$. $\boldsymbol{G}$, Normalized profiles. A single profile, corresponding to the traces in $\boldsymbol{F}$, is highlighted (black line). $\boldsymbol{H}$, Pooled values from $\boldsymbol{G}$ and linear fit.
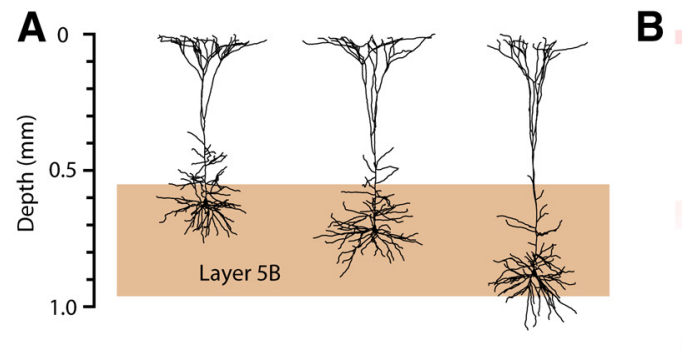

D
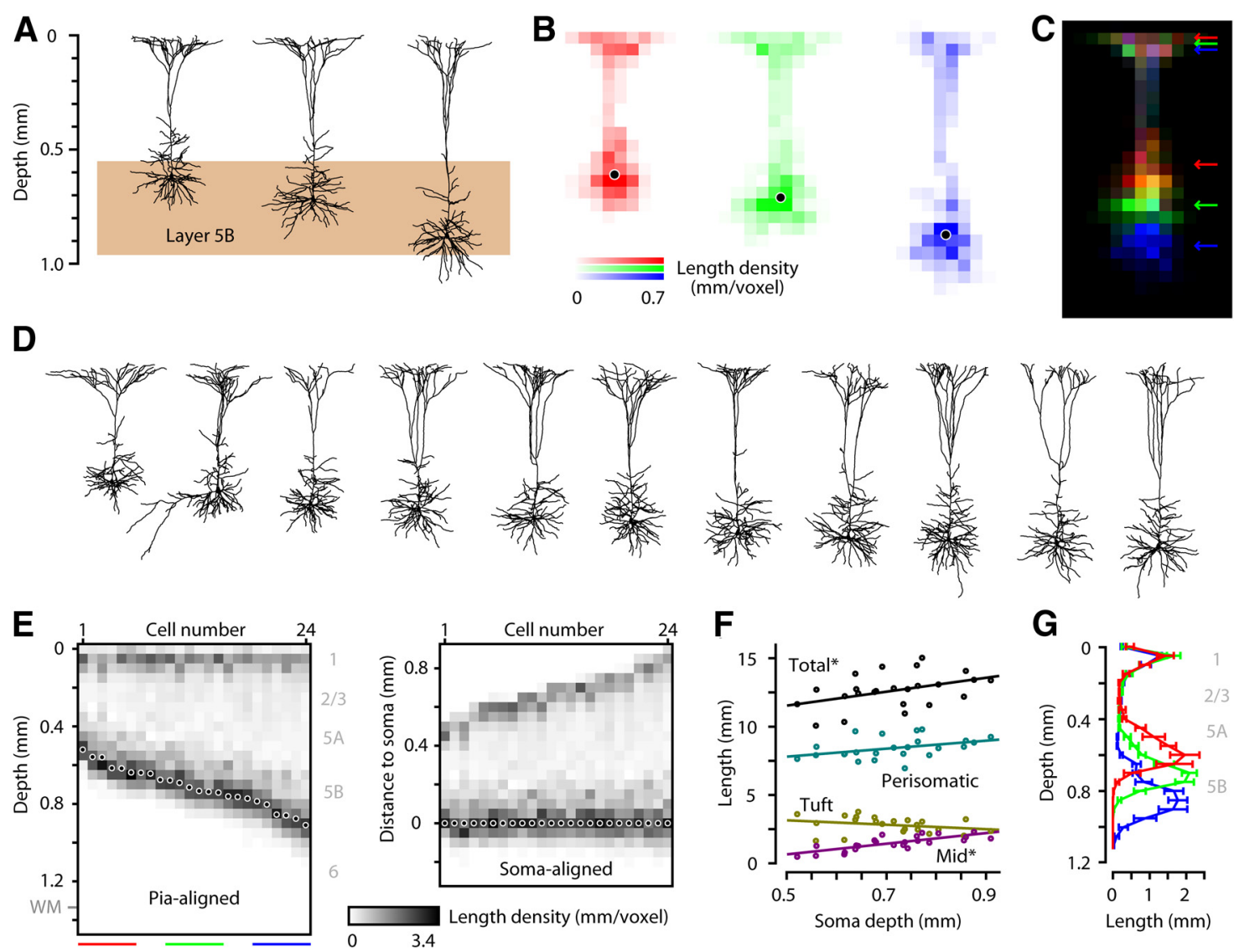

G

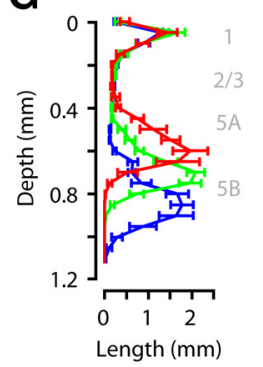

Figure 4. Dendritic morphology of M1-CSP is mostly independent of soma depth. $\boldsymbol{A}$, Three examples of 3D-reconstructed CSP neurons at upper, middle, deep depths in band (2D projections). $\boldsymbol{B}$, Length density maps for each of the example neurons. $\boldsymbol{C}$, Overlay of example maps separated by color, illustrating overlap in layer 1 , but differential coverage in deep layers. $\boldsymbol{D}$, Additional examples of 3D-reconstructed CSP neurons ( $n=11)$, ordered by soma depth and aligned to pia (2D projections; scale as in $A$ ). $E$, Vertical length density profiles for $n=24$ CSP neurons, ordered by soma depth, aligned to pia (left panel); aligned to soma (right panel). Colored bars under the plot indicate the three laminar regions used to group neurons as upper (red), middle (green), and lower (blue) CSP in subsequent analysis $(\boldsymbol{F}) . \boldsymbol{F}$, Relationship between soma depth and length density, for the total dendritic arbors, and separated into perisomatic, tuft, and intervening (mid) dendritic compartments. * Significant depth dependence. $\boldsymbol{G}$, Group analysis of upper, middle, and deep CSPs, grouped by soma depth as indicated in $\boldsymbol{D}$. 
A

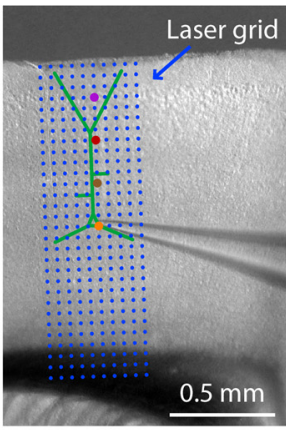

C

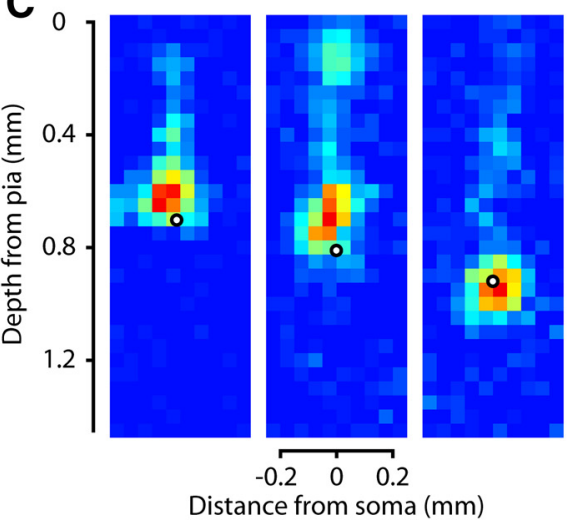

Laser

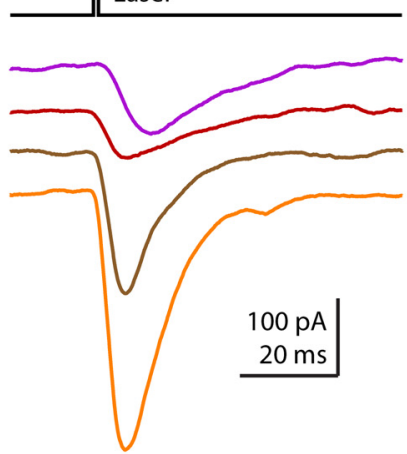

D

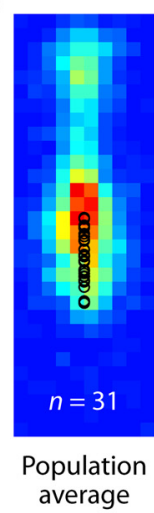

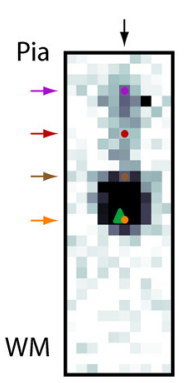

B

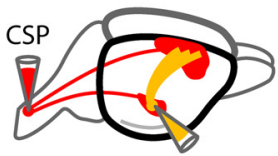

AAV-ChR2-Venus

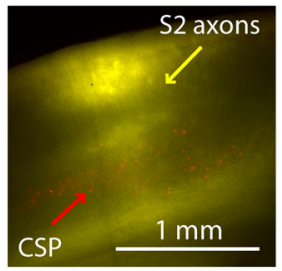

E

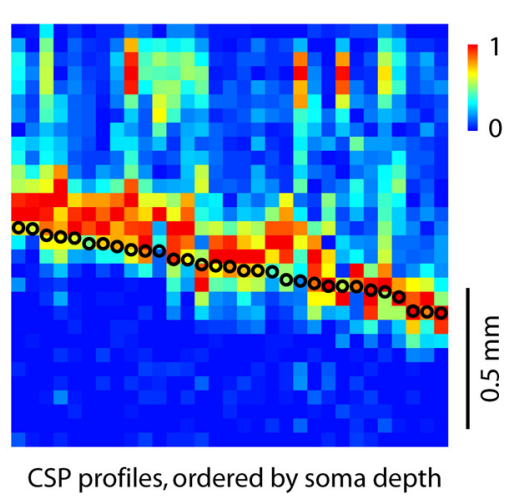

Figure 5. S2 axons innervate M1-CSP perisomatically and along apical dendrites, except in layer 1. $\boldsymbol{A}$, sCRACM technique. Left, Brightfield image of motor cortex and recording pipette, with overlay indicating laser photostimulation grid (array of blue dots with 30 rows, 10 columns, $50 \mu \mathrm{m}$ spacing). Stimulation sites along a selected column are indicated with colored circles. Middle, Example traces with colors corresponding to the stimulation sites in the column highlighted at left, ranging from superficial (purple) to deep (orange). Right, sCRACM map: the integral of each trace is converted to pixel intensity. Arrows point to the pixels corresponding to the four example traces. $B$, Schematic showing injection paradigm (left), and image of $S 2$ axons (yellow) in M1, with labeled CSPs (red). C, S2 to M1: three example maps; black circles represent CSP soma position. $\boldsymbol{D}$, Average sCRACM map ( $n=31$ neurons). $\boldsymbol{E}$, Input profiles by soma depth. Each CSP neuron's sCRACM map was converted to a single vertical profile (vector), and the collection of profiles was pooled and ordered by soma position.

cord with retrograde tracer. Subsequently, in M1 slices we recorded from labeled CSPs and obtained sCRACM maps of input from the ChR2-expressing thalamocortical (TC) axons. On average, and similar to S2, VL provided strong perisomatic input to CSPs (Fig. 6A). Unlike S2, VL axons also innervated both the distal-most apical tuft region in layer 1 and a distinct zone corresponding to the proximal apical arbor (layers 4 and 5A). Input to dendrites in layer $2 / 3$, on the other hand, was much weaker than to those in neighboring layers. This pattern was consistent across soma depths (Fig. 6B). For deep CSP, the layer 4/5A input formed a distinct band, although for shallower CSP this zone was confluent with the perisomatic input. Comparing the average input profiles from S2 and VL revealed a triply complementary, interdigitating pattern (Fig. $6 C$ ). These results suggest that CSPs can integrate inputs from S2 and VL, with potential for convergence onto the same dendritic branches in the perisomatic but not apical subarbors.

Next, we examined corticocortical input from secondary motor cortex (M2, located in the anterior frontal cortex). For this, we injected M2 with AAV-ChR2-Venus and spinal cord with retrograde tracer. Subsequently, in M1 slices, we recorded from labeled CSPs and obtained sCRACM maps of input from the ChR2-expressing M2 axons. On average, and again similar to S2, M2 provided strong perisomatic input (Fig. 6D). Unlike S2, M2 axons provided input to the apical tufts of CSPs in a depthdependent manner (Fig. 6E). Specifically, the relative amplitude of M2 input to the distal-most apical tuft $(0-275 \mu \mathrm{m}$ below the pia; see Fig. $6 E$, arrows) fell as a function of soma depth (Fig. $6 F$ ).
The data points were fit by a straight line with negative slope $\left(R^{2}\right.$ $=0.55 ;-7.6 \mathrm{~mm}^{-1}[-10.9$ to $\left.-4.3,95 \% \mathrm{CI}] ; p=0.00013\right)$. Applying this analysis to S2 inputs showed no depth dependence $\left(R^{2}=0.12 ;-1.5 \mathrm{~mm}^{-1}[-6.6\right.$ to $\left.3.7,95 \% \mathrm{CI}] ; p=0.57\right)$. These results suggest that, whereas CSPs at different depths receive S2 inputs in similar patterns, there is a substantial depth dependence for M2 inputs, with shallower but not deeper CSPs receiving strong M2 input in their distal apical tufts, relative to the perisomatic input. The depth dependence of this subcellular pattern could reflect greater perisomatic input to deeper CSPs, or less input to their distal tufts, or a combination of both. Furthermore, decreased distal tuft input could reflect fewer or weaker synapses, as well as greater dendritic attenuation for deeper CSPs. Overall, this subcellular pattern of input from M2 to M1-CSPs resembles the pattern seen for input from $\mathrm{M} 1$ to layer $5 \mathrm{~B}$ pyramidal neurons in S1 (Petreanu et al., 2009).

Next, we examined callosal input from homotypical, contralateral M1. For this, we injected M1 contralateral to our recording sites with AAV-ChR2-Venus, and spinal cord with retrograde tracer. Subsequently, in M1 slices, we recorded from labeled CSPs and obtained sCRACM maps of input from the ChR2-expressing callosal axons. Inputs occurred primarily at perisomatic sites, with additional input detected along the proximal and mid-apical dendrite but comparatively little in the distal tufts in layer 1 (Fig. $6 G$ ). In overall form, this map pattern most resembled that seen for inputs from S2. A subtle but significant difference, however, concerned a small upward offset in the location of the perisomatic input relative to the soma. Specifically, 
A

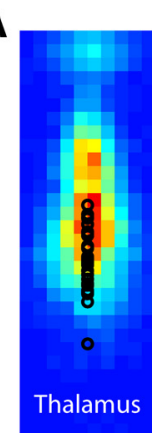

D

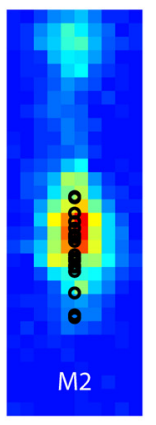

G

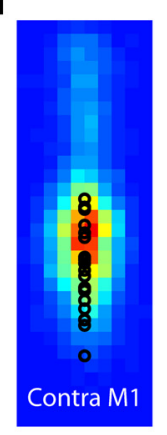

K

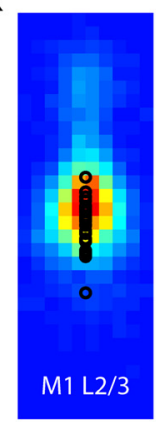

B

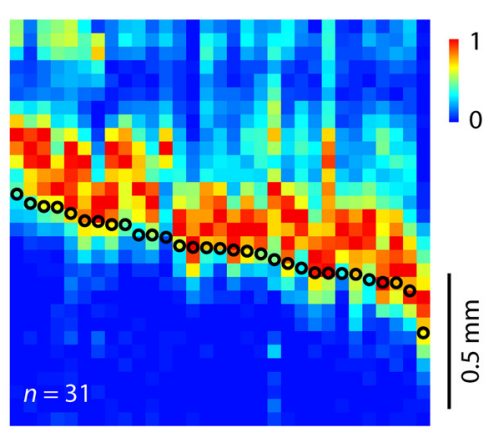

E

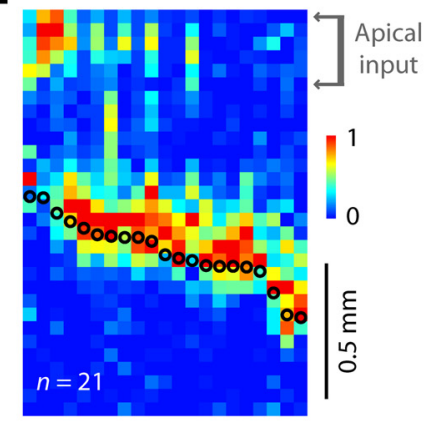

H

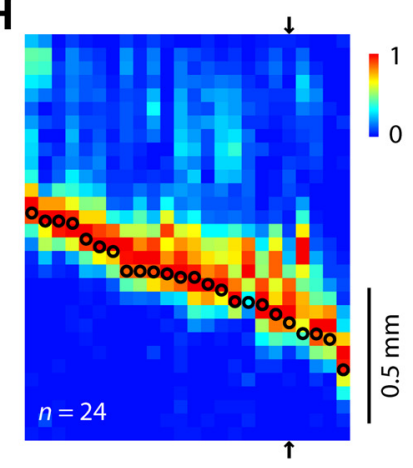

L
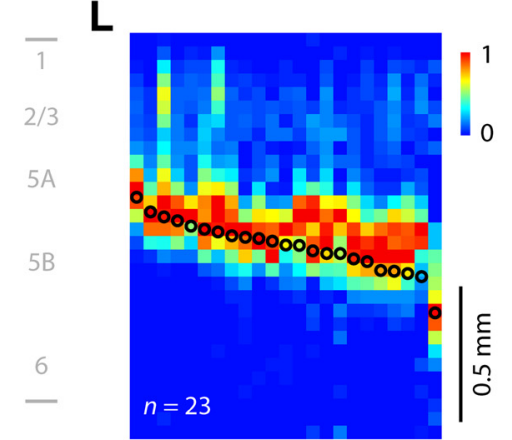

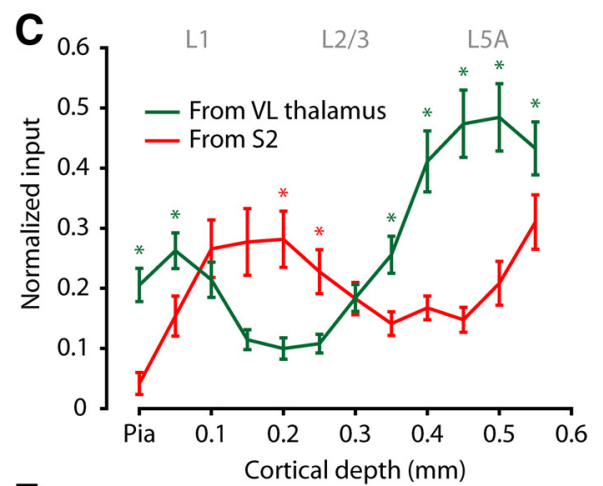

F

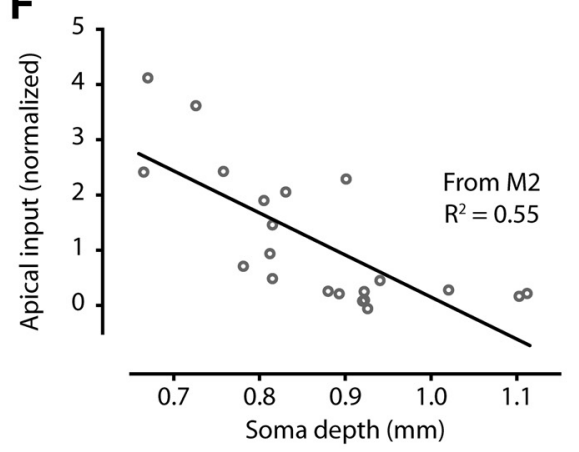

J
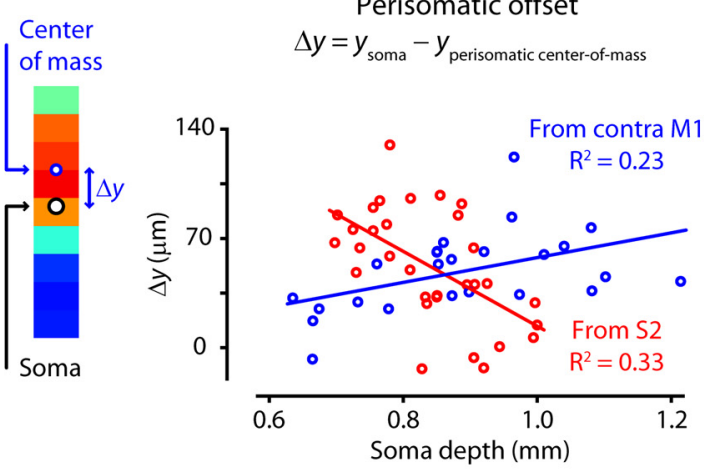

M

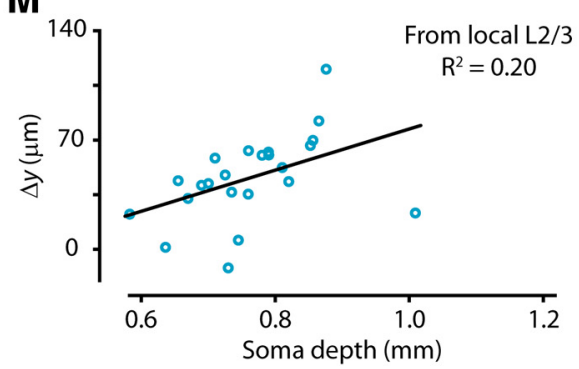

Figure 6. Axons from four other sources innervate M1-CSPs in source-specific subcellular input patterns. $\boldsymbol{A}$, Average sCRACM map of thalamic input to M1-CSPs ( $n=31$ neurons). $\boldsymbol{B}$, Input profiles by soma depth. Each CSP neuron's sCRACM map was converted to a single vertical profile (vector), and the collection of profiles was pooled and sorted by soma position. C, Comparison of thalamic (green) and S2 (red) input to the apical arbor (average across 31 neurons for each source \pm SEM). *Statistically significant difference ( $p<0.05$, Wilcoxon rank-sum test). $\boldsymbol{D}$, Average sCRACM map of M2 input to M1-CSPs $(n=21$ neurons). $\boldsymbol{E}$, Input profiles by soma depth. $\boldsymbol{F}$, Plot of apical input versus soma depth. Apical input was calculated as the sum over the top $275 \mu \mathrm{m}$ of each map (region indicated in $\boldsymbol{E}$ ). Line indicates linear regression. G, Average sCRACM map of contralateral M1 input to M1-CSPs ( $n=24$ neurons). $\boldsymbol{H}$, Input profiles by soma depth. $\boldsymbol{I}$, Illustration of center-of-mass calculation. A zoomed-in view of the perisomatic rows from a single profile (black arrows in $\boldsymbol{H}$ ) is shown. The perisomatic offset $(\Delta y)$ is the soma depth minus the center of mass of the perisomatic input (over the range $\pm 225 \mu \mathrm{m}$ from the soma). J, Perisomatic offset versus soma depth. Blue represents input from contralateral M1. Red represents input from S2. Lines indicate linear regressions. $K$, Average sCRACM map of layer $2 / 3$ inputs to M1-CSPs ( $n=23$ neurons). $L$, Input profiles by soma depth. $\boldsymbol{M}$, Location of perisomatic input from layer $2 / 3$ relative to soma position, plotted as a function of soma depth. For each profile, the input depth was calculated as the center of mass across the perisomatic pixels. 
A

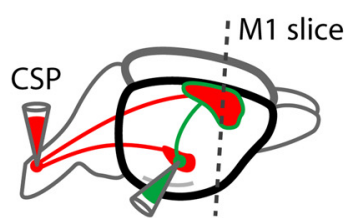

Retrograde tracer in S2

D

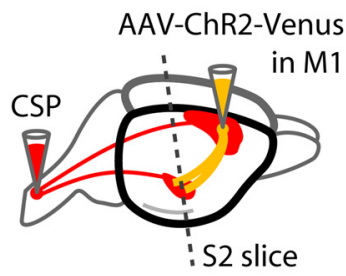

G
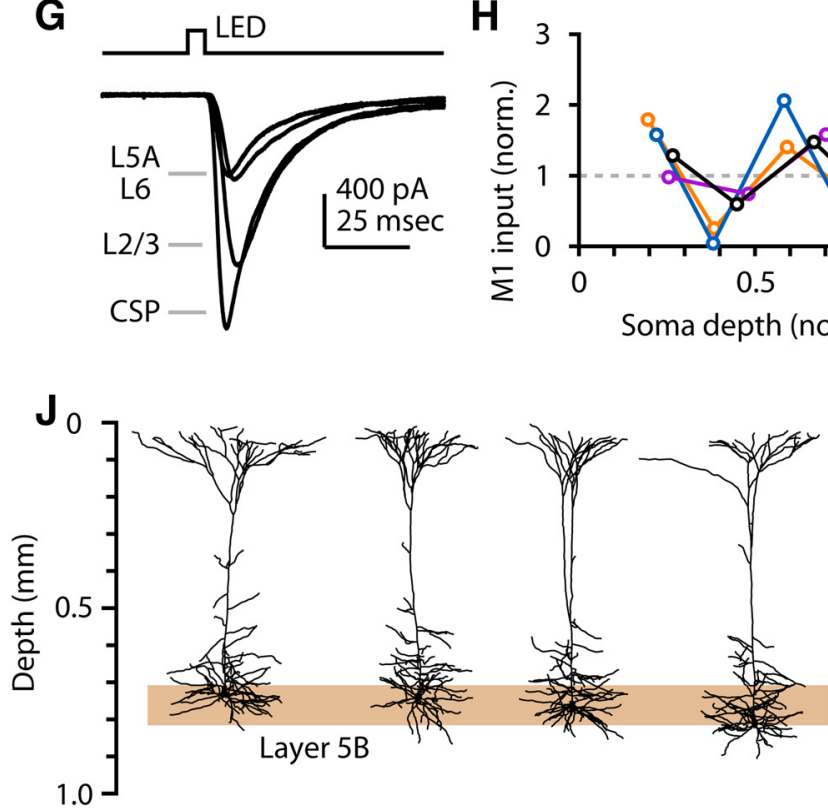

B

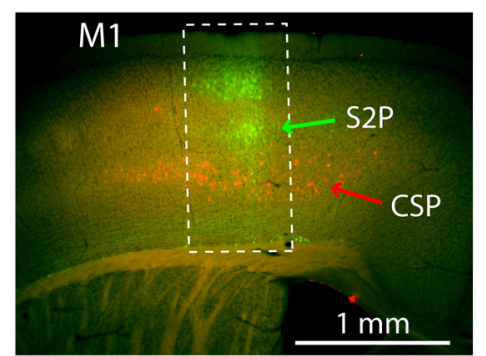

$\mathbf{E}$

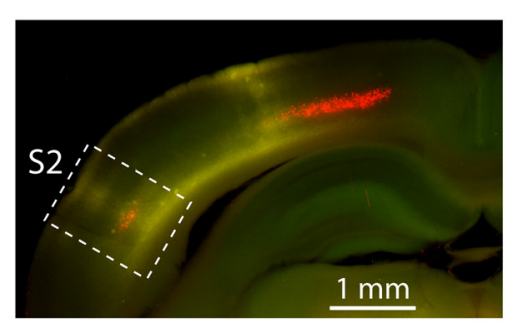

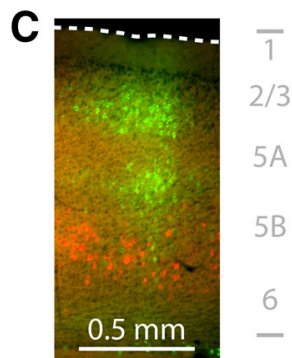

$\mathbf{F}$

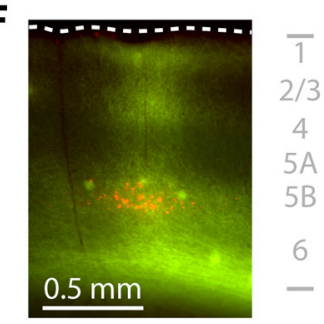

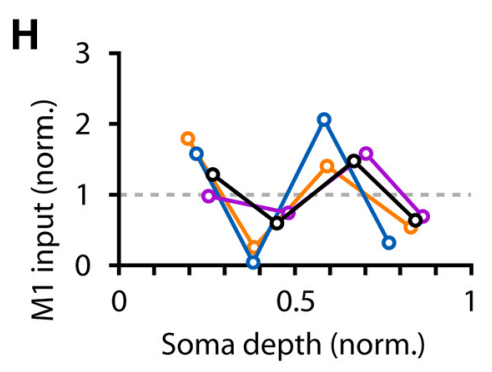

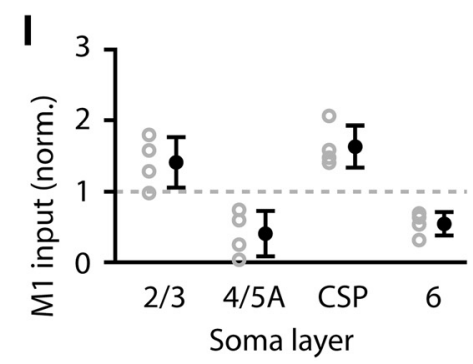

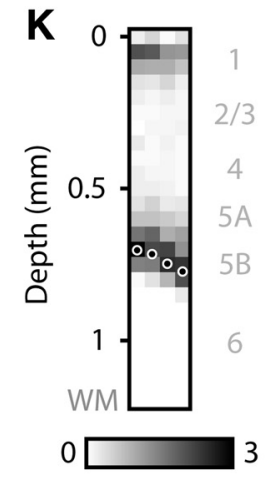

Length density ( $\mathrm{mm} /$ voxel)

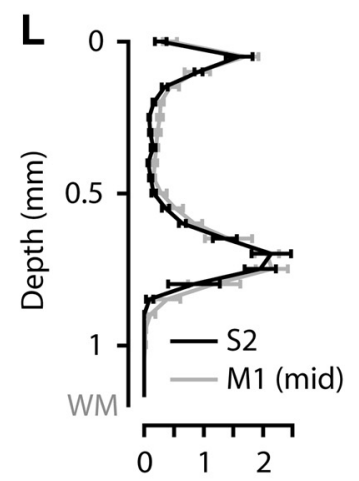

Length (mm)

Figure 7. M1 axons monosynaptically excite $\$ 2$ neurons across all layers. $A$, Injection paradigm. Retrograde tracer injections were made in the spinal cord and in $S 2$, and $M 1$ slices were examined for labeling of CSP and S2-projecting corticocortical (S2P) neurons. B, Low-magnification epifluorescence image showing retrograde labeling pattern in M1 slice (CSP red, S2P green). C, Highermagnification view of the labeling pattern. $\boldsymbol{D}$, Injection paradigm. Retrograde tracer was injected in spinal cord, AAV-ChR2-Venus was injected in M1, and S2 slices were prepared to visualize and photostimulate M1 axons while recording from CSP and other S2 neurons. $\boldsymbol{E}$, Low-magnification epifluorescence image showing M1 axons (yellow) and CSPs (red) in S2.F, Higher-magnification image showing M1 axons (yellow) overlapping with S2-CSPs (red; arrow). G, Example traces. Wide-field illumination with a gated LED was used to photostimulate the ChR2-expressing M1 axons while recording from $\mathbf{2} 2$ neurons in different layers (as indicated). $\boldsymbol{H}$, Laminar profiles of $\mathrm{M} 1$ input to $S 2$. Each colored line indicates a profile obtained in a single slice. Data were normalized to the mean value per profile. Soma depth was normalized: $0=$ pia; $1=$ white matter. The black profile corresponds to the example traces in $\mathbf{G}$. I, Same experiments as in the previous panel, but with the values (gray) pooled across profiles by layer and averaged (black, mean \pm SEM). J, Four 3D-reconstructed S2-CSP neurons (2D projections) from different depths in layer 5B (shaded region). $\boldsymbol{K}$, Vertical length density profiles for the neurons in $J$, aligned to the pia. $L$, Group average of the length density as a function of cortical depth for S2-CSP (black) and for a subset of M1-CSP (gray, $n=$ 4) at matching soma depths.

this "perisomatic offset" appeared to increase with CSP soma depth for contralateral M1 inputs (Fig. $6 H$ ), the opposite of the trend for S2 inputs (Fig. 5E). To quantify this, we calculated the perisomatic offset as the distance between the soma and the center of mass of perisomatic inputs (within $\pm 200 \mu \mathrm{m}$ of the soma) (Fig. 6I). This analysis confirmed that, although the average perisomatic offset was similar $(\sim 50 \mu \mathrm{m})$ for both sources (contra M1: $48.8 \pm 5.3 \mu \mathrm{m}$; S2: $51.8 \pm 6.6 \mu \mathrm{m})$, the depth dependence was opposite: for contralateral M1 input, the offset increased with soma depth $\left(R^{2}=0.22 ; 79.5 \mu \mathrm{m} / \mathrm{mm}[14.2\right.$ to $144.7,95 \% \mathrm{CI}]$; $p=0.019)$, whereas for S2 input, the offset decreased with soma depth $\left(R^{2}=0.33 ;-239.6 \mu \mathrm{m} / \mathrm{mm}[-370.3\right.$ to $-108.8,95 \% \mathrm{CI}]$; $p=0.0008)$.

Finally, we examined local input from layer $2 / 3$, a particularly strong source of interlaminar excitation to CSPs in M1 (Anderson et al., 2010). For this, we transfected M1 layer 2/3 pyramidal 
A

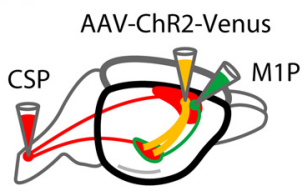

B

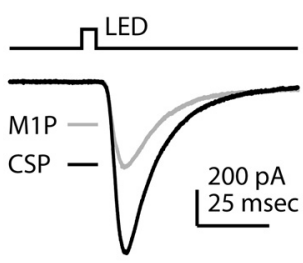

D

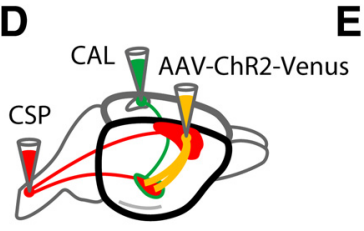

C

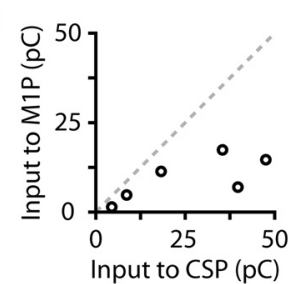

$\mathbf{F}$

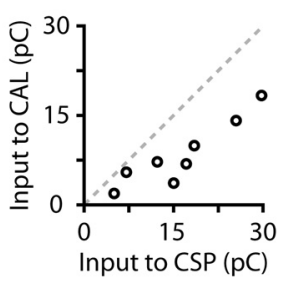

Figure 8. M1 axons excite layer $5 B$ neurons in $S 2$ with a bias toward CSP. $A$, Labeling paradigm. Retrograde tracers were injected into the spinal cord and M1 cortex to label CSP and M1-projecting (M1P) neurons, and AAV-ChR2-Venus was injected into M1. $B$, Example traces, recorded from an adjacent pair of CSP and M1P neurons in layer 5B. C, Group data, showing pairwise responses from CSP and M1P neurons. D, Labeling paradigm. Retrograde tracers were injected into the spinal cord and contralateral S2 cortex to label CSP and CAL neurons, and AAV-ChR2 was injected into M1.E, Example traces, recorded from an adjacent pair of CSP and CAL neurons in layer $5 B . F$, Group data, showing pairwise responses from CSP and CAL neurons.

neurons via in utero electroporation, a technique that exploits the inside-out developmental sequence of excitatory neocortical neurons (Saito and Nakatsuji, 2001; Tabata and Nakajima, 2001, 2008). We later injected the adult spinal cord with retrograde tracer. Subsequently, in M1 slices, we recorded from labeled CSPs and obtained sCRACM maps of input from the ChR2-expressing local axons of layer $2 / 3$ pyramidal neurons. In this case, we sampled primarily from CSPs in the upper half of layer $5 \mathrm{~B}$, as those in the lower half receive little or no layer 2/3 input (precluding sCRACM mapping) (Anderson et al., 2010). The population average map (Fig. 6K) and profiles (Fig. $6 L$ ) appeared similar to those arising from contralateral $\mathrm{M} 1$, with most of the input in the perisomatic region. The perisomatic offset increased with soma depth $\left(R^{2}=0.20 ; 131.9 \mu \mathrm{m} / \mathrm{mm}[11.8\right.$ to $251.9,95 \% \mathrm{CI}] ; p=$ $0.033)$, similar to the offset described for layer $2 / 3$ input in $S 1$ (Petreanu et al., 2009). These results suggest that M1-CSPs receive callosal inputs from contralateral M1 and local inputs from ipsilateral layer $2 / 3$ in a similar subcellular pattern. Comparably, in $\mathrm{S} 1$, local and callosal projections from layer 2/3 display a common pattern of laminar excitation at the cellular level (Petreanu et al., 2007); our findings extend this to M1 in the subcellular domain.

From these subcellular mapping experiments, we conclude the following: (1) each upstream source of input to M1 examined here monosynaptically excited CSP neurons; (2) the five input pathways share a similar overall (i.e., average, albeit with considerable cell-to-cell variability) pattern of targeting primarily perisomatic sites, usually with additional inputs detected along apical locations; (3) each input source showed pathway-specific features (e.g., thalamic input to distal tuft) that distinguished it to varying degrees from the S2 input pattern; and (4) overall, the contralateral M1 pattern most closely resembled the S2 input pattern.

\section{M1 axons monosynaptically excite S2 neurons across all layers}

The following experiments examine connectivity in the reverse direction, from M1 to S2, again focusing on the innervation of CSPs in the downstream area. We used a similar approach as for the S2 $\rightarrow$ M1 studies, except that the thinness of layer 5B (and accordingly of CSP labeling) in S2 largely obviated depth-dependent analyses. As a starting point, to identify and localize the M1 subregion containing S2-projecting neurons, we used the following labeling strategy (Fig. $7 A$ ): we injected tracer into the spinal cord as usual and injected tracer (of another color) into S2. In subsequently prepared M1 slices, we observed retrogradely labeled S2-projecting neurons in $\mathrm{M} 1$, overlapping with the S2recipient zone (Fig. $7 B, C$ ). These labeled neurons were never double-labeled with the spinal tracer, and were located across multiple M1 layers, except layer 1 (Fig. 7C). Injection of AAV-ChR2-Venus into M1 (Fig. 7D) resulted in labeling of M1 axons in S2, overlapping with the labeled CSPs (Fig. 7E,F); axons were present in all layers, including layer 1 , with prominent peaks in layer $2 / 3$ and layer 5 . This gave a more "columnar" appearance for the axons in S2 compared with those in neighboring S1, where axon labeling was strongest in layer 1, as previously observed (Petreanu et al., 2009; Kinnischtzke et al., 2014). Unlike in $M 1$, where axons from $S 2$ innervated a subset of the relatively wide CSP band (Fig. $3 A-D$ ), here the axons from M1 extended fully over the S2-CSP field (width in coronal plane, mean \pm SEM, $0.78 \pm 0.07 \mathrm{~mm}, n=10$ animals).

We recorded from S2 neurons across multiple layers in each slice and sampled the excitatory responses to photostimulation of the ChR2-expressing M1 axons (Fig. 7G). In this experiment, we did not record blindly from layer $5 \mathrm{~B}$ neurons but targeted recordings to CSPs as a way to ensure the laminar location of the recording. We analyzed the relative amplitudes of responses across layers to generate laminar profiles of M1 input to these S2 neurons (Fig. $7 \mathrm{H}, \mathrm{I}$ ). This analysis showed that M1 axons made monosynaptic contacts onto S2 neurons across all layers, but with relatively strong innervation for inputs to layer $2 / 3$ neurons and also to S2-CSPs, and much weaker input to neurons in layers $5 \mathrm{~A}$ and 6 (Fig. $7 \mathrm{H}, I$ ). This pattern thus appears qualitatively similar, but quantitatively different, compared with the pattern in the reverse $\mathrm{S} 2 \rightarrow \mathrm{M} 1$ direction.

Because dendritic morphology of M1-CSP was largely independent of soma depth, and because the CSP band is only $\sim 100$ $\mu \mathrm{m}$ thick in S2, we expected that S2-CSP would share similarly characteristic morphology. To confirm this, we imaged dye-filled S2-CSPs, reconstructed their dendritic arbors (Fig. 7J) and analyzed these structures by converting them to maps of dendritic length density. As in M1, S2-CSPs were characterized by high dendritic length density in the basal/perisomatic compartment and in the apical tuft. These regions were linked by a long apical trunk with minimal branching. Vertical length density profiles (Fig. $7 K$ ) showed that the shape of the dendritic arbor did not vary with soma depth. Dendritic length (overall mean $\pm \mathrm{SD}$ : $1.14 \pm 0.04 \mathrm{~cm}$ ) was distributed between the apical tuft and perisomatic compartment in similar proportions as seen in M1 (ratio tuft/perisomatic, mean \pm SEM, S2: $0.37 \pm 0.04, \mathrm{M} 1: 0.33 \pm 0.02$, $p=0.49)$. To compare with M1-CSP morphology, for each S2CSP neuron, we selected an M1-CSP neuron at the closestmatching cortical depth (soma-to-pia distance). The vertical profile of length density for S2-CSP overlapped extensively with that for M1-CSP at matching soma depth $(n=4)$ (Fig. $7 L)$. From 
A

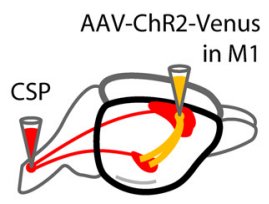

E

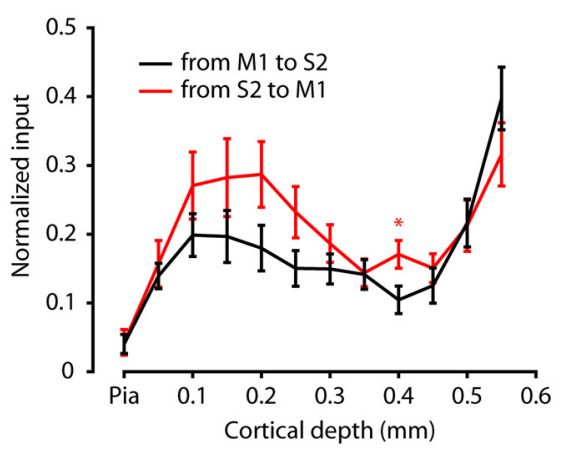

B

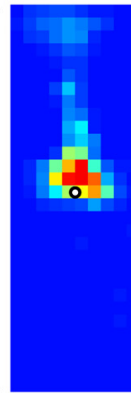

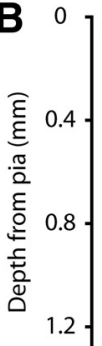

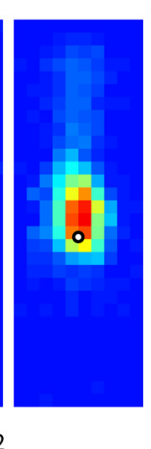

soma ( $\mathrm{mm}$ )
$\mathbf{F}$

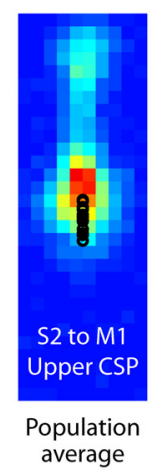

C
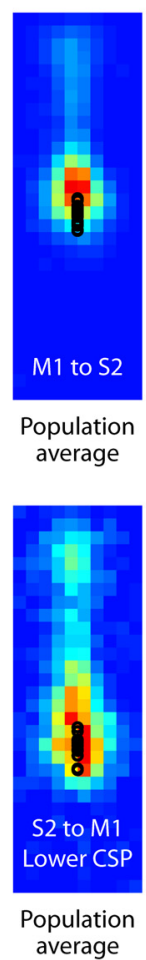

D

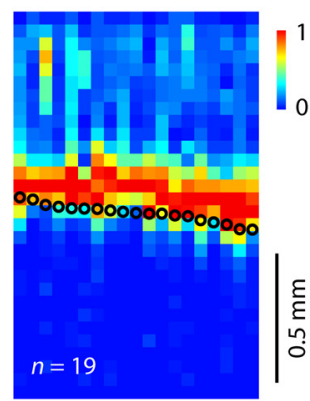

CSP profiles ordered by soma depth

G

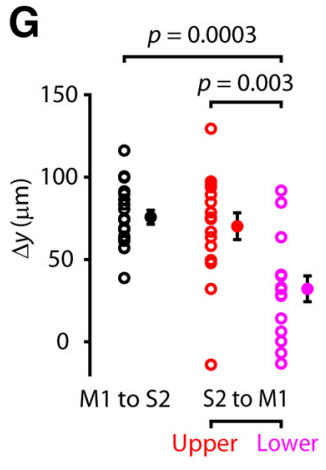

Figure 9. M1 axons innervate S2-CSP dendrites much like the S2 $\rightarrow$ M1-CSP subcellular input pattern. $\boldsymbol{A}$, Labeling paradigm. Retrograde tracer was injected into the spinal cord to label CSP neurons, and AAV-ChR2-Venus was injected into M1. $\boldsymbol{B}$, Three example maps; black circles represent CSP soma position. C, Average sCRACM map of M1 input to S2-CSPs ( $n=19$ neurons). $\boldsymbol{D}$, Input profiles by soma depth. Each CSP neuron's sCRACM map was converted to a single vertical profile (vector), and the collection of profiles was pooled and ordered by soma position. $\boldsymbol{E}$, Comparison of input (average \pm SEM for each source) to the apical arbor from M1 to S2-CSP (black, $n=19$ neurons) and from S2 to M1-CSP (red, $n=31$ neurons). A single bin was significantly different and is indicated with an asterisk ( $p<0.05$, Wilcoxon rank-sum test). $\boldsymbol{F}$, Average sCRACM map of $S 2$ input to upper M1-CSPs ( $n=16$ neurons; left), and to lower M1-CSPs ( $n=15$ neurons, right). $\boldsymbol{G}$, Location of interareal perisomatic input relative to soma position. For each profile, the input depth was calculated as the center of mass across the perisomatic pixels. The offset for M1 to S2-CSPs differs compared with S2 input to lower M1-CSPs, but not compared with upper M1-CSPs.

these morphological analyses, we conclude that CSP neurons in the two areas share the same characteristic form comprising a large perisomatic compartment with $\sim 65 \%$ of the total dendritic length and a smaller but substantial apical tuft compartment (primarily in layer 1 ) with $\sim 25 \%$, linked by an intervening apical segment the length of which is proportional to the cortical depth of the particular CSP.

\section{M1 axons excite layer 5B neurons in S2 with a bias toward CSP}

Next, we investigated whether the strong input from M1 to S2CSPs reflected cell-type specific innervation, by recording from S2-CSPs and comparing their responses to neighboring pyramidal neurons in layer 5B identified as M1-projecting corticocortical neurons. For this, in addition to injecting M1 with AAV-ChR2, we also injected retrograde tracers (different colors) into the spinal cord and M1 (Fig. 8A). We then selected a pair of neighboring pyramidal neurons of each type (CSP and M1-projecting) for sequential recordings (Fig. $8 B$ ). We observed no double-labeled neurons, confirming that these are nonoverlapping projection classes. Responses in S2-CSP and M1-projecting neurons in layer $5 \mathrm{~B}$ consistently showed stronger input to S2-CSP neurons $(p=$ 0.03; median ratio of M1P/S2-CSP: 0.40) (Fig. 8C). To confirm and extend these findings, we repeated this experiment but recorded from CAL neurons in layer $5 \mathrm{~B}$ of S2, labeled by injecting contralateral S2 instead of ipsilateral M1 (Fig. 8D). Again, comparing responses in S2-CSP and neighboring CAL neurons to M1 input, we observed consistently stronger input to S2-CSP neurons $(p=0.008$; median ratio of CAL/S2-CSP: 0.55) (Fig. $8 E, F)$.
These results thus indicate that M1 axons preferentially target CSP neurons over other types of projection neurons in layer $5 \mathrm{~B}$ of S2. Furthermore, M1 axons monosynaptically excite S2-CSPs, forming a basis for a long-range corticocortical $\rightarrow$ corticospinal pathway, and they also monosynaptically excite M1-projecting neurons in S2, forming a basis for reciprocal coupling (i.e., a feedback loop) between the two areas; both of these long-range pathways mirror those described above for M1 neurons.

\section{M1 axons innervate S2-CSP dendrites much like the S2 $\rightarrow$ M1-CSP subcellular input pattern}

We performed the same sCRACM analysis used previously in M1 to examine the subcellular targeting of S2-CSP by axons from M1 (Fig. 9). For this, we injected M1 with AAV-ChR2-Venus and spinal cord with retrograde tracer (Fig. 9A). Subsequently, in S2 slices, we recorded from labeled CSPs and obtained sCRACM maps of input from the ChR2-expressing M1 axons (Fig. 9B). On average (Fig. 9C), M1 provided strong perisomatic input, with additional sites along the apical dendrites but few in the distal tufts (despite the presence of axons in layer 1). This general pattern closely resembled that observed for maps in the reciprocal direction, S2 $\rightarrow$ M1-CSPs. The laminar profiles of input in either direction across the apical dendrites of CSPs in both areas were highly similar (Fig. 9E). One difference was that, whereas the perisomatic offset was depth-dependent for input from S2 to M1-CSP (Fig. 6J), it was not for M1 to S2-CSP $\left(R^{2}=0.07 ; 147.5\right.$ $\mu \mathrm{m} / \mathrm{mm}[-120.4$ to $415.3,95 \% \mathrm{CI}] ; p=0.26)$. However, whereas layer $5 \mathrm{~B}$ in $\mathrm{M} 1$ is a relatively thick layer composed of upper and lower sublayers with distinct cellular and circuit-level 

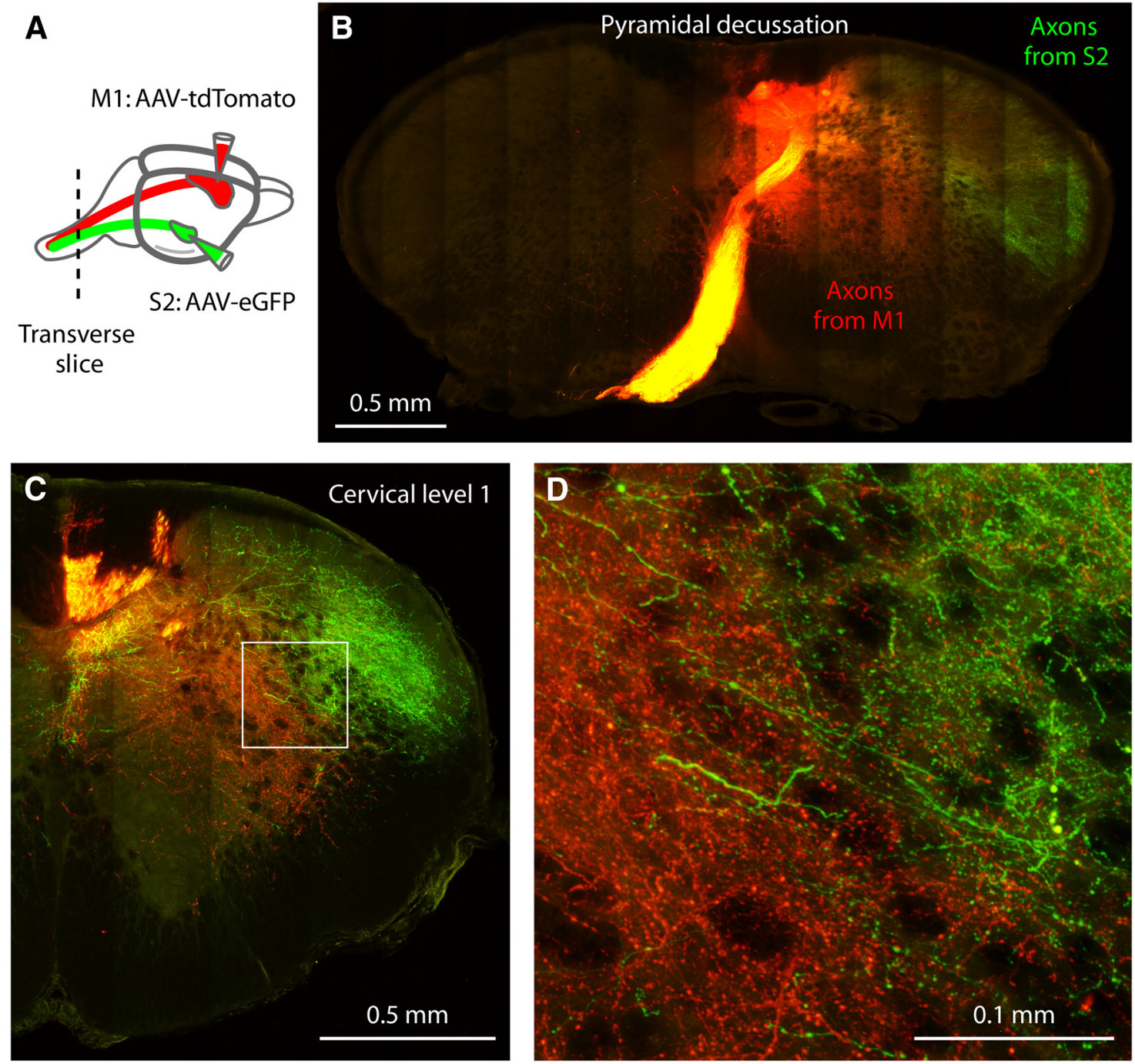

Figure 10. M1-CSP and S2-CSP axons differentially project to cervical spinal cord. A, Labeling paradigm. AAV-tdTomato was injected into M1, and AAV-eGFP was injected into S2. B, Two-photon image taken at the level of the pyramidal decussation. Red: M1 axons; green: $\$ 2$ axons. The axons travel together in the decussation (their overlap appearing as yellow). In the gray matter of the spinal cord, the M1 axons are distributed mostly medially and the $\$ 2$ axons mostly laterally; both are mostly in the dorsal half of the cord. C, Image from same animal at the level of C1. D, Higher-magnification view of the region of interest marked by the box in the preceding panel.

properties (Yu et al., 2008; Anderson et al., 2010; Hooks et al., 2013), layer 5B in S2 is a much thinner layer and S2-CSPs were accordingly distributed over a relatively narrow range of cortical depth. Qualitatively, the perisomatic offset appeared most similar to that of their M1 counterparts in the upper part of layer 5B. This was borne out both in the average maps (Fig. $9 E$ ) and group comparisons (Fig. 9F) of the upper and lower halves of the M1CSP sample (M1 to S2-CSP, $n=19: 75.7 \pm 4.3 \mu \mathrm{m}$; S2 to upper M1-CSP, $n=16: 70.2 \pm 8.1 \mu \mathrm{m}$; S2 to lower M1-CSP, $n=15$ : $32.2 \pm 7.9 \mu \mathrm{m})$. Overall, these analyses of the subcellular distribution of M1 inputs to S2-CSPs suggest that the mutual connections are similarly patterned and furthermore that in $\mathrm{M} 1$ it is the CSPs in upper layer 5B that most exhibit this similarity.

\section{M1-CSP and S2-CSP axons differentially project to cervical} spinal cord

Last, we explored the spinal trajectories of CSP axons originating from M1 and S2. For this, we injected forelimb-M1 and S2 with AAV carrying green or red fluorescent proteins (Fig. 10A). In 2 mice, M1/S2 CSPs were labeled green/red, respectively, and in 2 mice, they were labeled red/green; results were similar with either combination. Transverse slices of the entire cervical spinal cord were prepared. Axons from both sources were observed to arborize primarily at cervical spinal cord levels, the segments containing motor neurons of the forelimb muscles (Bachmann et al., 2014), thus confirming accurate localization of forelimb-M1. We selected multiple sections distributed across cervical spinal levels for high-resolution two-photon microscopy. CSP axons from $\mathrm{M} 1$ and S2 were intermingled in the pyramidal decussation (Fig. $10 B$ ) but then diverged considerably in the contralateral gray matter cord, with the terminal arbors from each cortical source targeting largely distinct but partially overlapping regions in the rostral cervical cord. This segregation was greatest rostrally, from cervical levels $\mathrm{C} 1-\mathrm{C} 3$, where there was some overlap but the S2 axons branched mostly in the dorsal horn, whereas M1 axons branched mostly in the middle laminae (Fig. 10C,D), the latter pattern consistent with prior observations in rodents (Casale et al., 1988; Liang et al., 1991; Ghosh et al., 2009). At more caudal levels (C6-C8), there was less branching from either source, and also increasing overlap, chiefly in the superficial and middle regions of the cord (laminae 1-5). Within the white matter of the cervical cord, the CSP axons from M1 and S2 traveled almost 
entirely in the contralateral dorsal corticospinal tract. Axons from M1-CSPs and S2-CSPs were intermingled within this tract, similar to previous observations of nontopographic organization (i.e., intermingling) of forelimb and hindlimb CSP axons within the dorsal CST (Jeffery and Fitzgerald, 1999). These results indicate that the spinal trajectories of CSP axons from M1 and S2 are organized in a partly convergent and partly parallel manner: they both project to the middle layers of the cord, but M1-CSP axons extend more medially and ventrally, and S2-CSP axons ramify extensively in the most dorsal and lateral regions of the upper cervical cord, including layer 1 .

\section{Discussion}

We studied the circuit organization of the mutual connections of two CSP-containing areas, M1 and S2, focusing on characterizing the interareal corticocortical inputs to their CSPs. By recording from labeled CSPs and exploiting the preserved photoexcitability of ChR2-expressing axons in slices (Petreanu et al., 2007), together with methods for focally exciting synaptic terminals along these axons (Petreanu et al., 2009), we were able to analyze monosynaptic inputs from long-range axonal projections at cellular and subcellular (dendritic) resolution. Overall, we found that the mutual connections S2 $\rightarrow$ M1-CSP and M1 $\rightarrow$ S2-CSP closely resemble one another, albeit with several area-specific differences. Thus, the circuits of M1 and S2 are not only organized in parallel but are synaptically linked through interareal connections that directly innervate CSPs in each area.

Our results may help to elucidate the hierarchical relationships between $\mathrm{M} 1$ and $\mathrm{S} 2$ in rodents, and between these and other sensorimotor cortical areas (Fig. 11A). In rodent sensory cortex, axons in feedforward projections ("bottom-up," from lower- to higher-order areas) typically ramify in the middle layers (layers $2 / 3$ through $5 \mathrm{~A}$ ), whereas those in feedback projections ("topdown," from higher- to lower-order) ramify mostly in layer 1 and layer 5B (Coogan and Burkhalter, 1993). Recently, these anatomical patterns have been substantiated by optogenetic-electrophysiology methods assessing interareal monosynaptic excitatory synaptic connections onto postsynaptic neurons in the downstream area (Petreanu et al., 2009). Thus, M1 appears higher-order relative to S1, and lower-order relative to M2 (Petreanu et al., 2009; Mao et al., 2011; Hira et al., 2013; Hooks et al., 2013; Ueta et al., 2013, 2014). In particular, the subcellular innervation from M2 to M1CSPs closely resembles the top-down pattern previously found for the projection from vibrissal M1 to layer 5B pyramidal neurons in S1 (Petreanu et al., 2009). Although S2 is also usually considered higher order relative to S1 (Burton, 1986), recent evidence indicates that $\mathrm{S} 2$ is at a similar hierarchical level as S1, based on parallel thalamocortical projections to both areas from the ventral posterolateral nucleus, symmetric interareal connectivity, and similar sensory-evoked response latencies (Liao and Yen, 2008). Our findings are most consistent with the idea that $\mathrm{M} 1$ and S2 are at an approximately equivalent level because the innervation patterns were bidirectionally similar, including

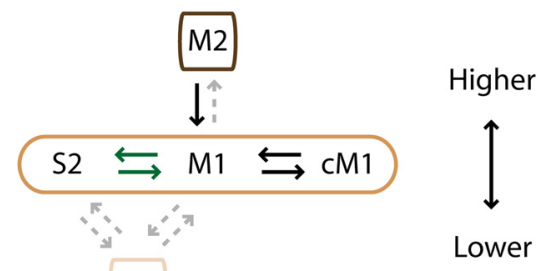

S1

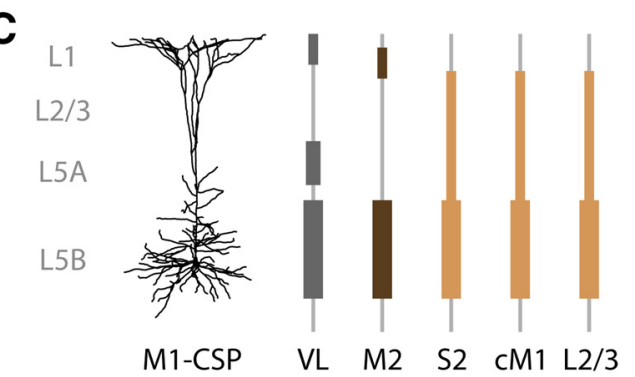

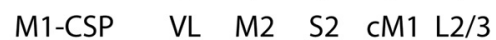

Figure 11. Schematics summarizing some of the findings. $\boldsymbol{A}$, Hierarchical relationships among sensorimotor areas. The approximate anatomical locations of M1, S2, M2, and S1 are depicted on the left, and the hierarchical relationships are depicted on the here; gray dashed arrows indicate known projections not studied here. $\boldsymbol{B}$, Interareal projections between M1 and S2. The presynaptic and postsynaptic neurons were distributed across multiple cortical layers (green-outlined pyramids). Corticospinal neurons Alloway, 2013). C, Subcellular innervation of M1-CSP dendrites by different afferent sources. The vertical positions and the widths of the shaded boxes show qualitatively the laminar location and relative strength of input. VL, Ventrolateral nucleus of thalamus.

monosynaptic excitation of both deeper-layer (e.g., CSPs) and upper-layer (e.g., layer 2/3) neurons (Fig. 11B). Supporting this interpretation, the M1 $\leftrightarrow$ S2 subcellular connectivity patterns also closely resembled the interhemispheric M1 $\leftrightarrow$ M1 connectivity pattern, and were distinct from the $\mathrm{M} 2 \rightarrow \mathrm{M} 1$ pattern (Fig. 11C). Furthermore, the laminar distribution of originating neurons, and the horizontally restricted, columnar innervation of mutual M1 $\leftrightarrow$ S2 connections most resemble the pattern of lateral connectivity between areas at the same level in the cortical hierarchy (Felleman and Van Essen, 1991).

The pattern of reciprocal connectivity was mostly but not exactly symmetric. Specifically, whereas S2 excited layer $5 B$ neurons in M1 independent of projection type, in the reverse direction, M1 $\rightarrow$ S2 input was biased approximately twofold toward CSP over neighboring corticocortical neurons. Despite this asymmetry, the finding that $\mathrm{M} 1 \rightarrow \mathrm{S} 2$ axons monosynaptically excite layer 5B neurons projecting back to $\mathrm{M} 1$, and that $\mathrm{S} 2 \rightarrow \mathrm{M} 1$ projections in turn excite layer $5 \mathrm{~B}$ neurons projecting back to $\mathrm{S} 2$, suggests that M1 and S2 are tightly and probably bidirectionally coupled at the cellular level.

M1 and S2 share additional circuit-level properties, albeit with area-specific features. Both areas receive corticocortical projections from S1 (see Introduction) and thalamocortical projections from the posterior $(\mathrm{PO})$ nucleus of the thalamus (Herkenham, 1980; Burton, 1986; Theyel et al., 2010; Pouchelon et al., 2014). However, the circuits of M1 and S2, although similar, are also differentiated in several ways. The S1 projections to M1 and S2 originate from anatomically separate subsets of layer $2 / 3$ neurons with distinct functional properties (see Introduction). The thalamic projections to M1 and S2 originate not only from PO but from additional nuclei that project to only one of the areas (e.g., projections to M1 from the ventrolateral nucleus and to S2 from the ventrobasal and medial geniculate nuclei) (Burton, 1986; Carvell and Simons, 1987; Pierret et al., 2000; Jones, 2007). Map- 
ping of the local circuits across layers has suggested these to be qualitatively similar but with area-specific quantitative differences (Hooks et al., 2011). Our findings together with these observations suggest a circuit organization for M1 and S2 in which both of these CSP-containing areas are intimately linked through reciprocal interareal connections, partly common inputs (from $\mathrm{S} 1$ and $\mathrm{PO}$ ), and partly common outputs (partially overlapping spinal cord trajectories).

These results may be relevant to the concept of a "sensorimotor interface" in cortical networks involved in sensory perception, decision-making, and motor control (Romo and Salinas, 2001). Sensory responses are often observed in M1 neurons and may depend on S2 in particular (Burton, 1986). Indeed, in a recent study in the rat, the $\mathrm{S} 2 \rightarrow \mathrm{M} 1$ projection was proposed to subdivide M1 into sensory-input and motor-output areas (Smith and Alloway, 2013). The converse possibility, that M1 $\rightarrow$ S2 projections endow S2 with "motor" properties, has not been functionally assessed but would be consistent with the M1 $\rightarrow$ S2 projections reported here.

A challenge in studying $\mathrm{M} 1 \leftrightarrow \mathrm{S} 2$ circuits is the complexity of somatotopic representations within each area. Here, we localized forelimb regions of $\mathrm{M} 1$ and S2 based on application of standard criteria (see Materials and Methods), the presence of labeled CSPs in both areas following injections of retrograde tracer into the cervical spinal cord, and reciprocal anterograde innervation. Further studies will be required to dissect these interareal circuits at finer levels, such as the adductor-abductor subregions of forelimb M1 (Harrison et al., 2012), or the various body part representations in S2 (Burton, 1986; Carvell and Simons, 1986), and to link response properties of neurons in these different subregions to cell-specific synaptic circuits.

The M1 $\leftrightarrow$ S2 circuits delineated in this study have potential implications for disease-related studies. In particular, the monosynaptic corticocortical $\rightarrow$ corticospinal connections may be a substrate for plasticity following manipulations (e.g., lesions, stimulation) affecting either cortical area. Indeed, a recent study noted an increase of S2-CSP axons in the spinal cord in an M1 stroke model (Bachmann et al., 2014). Furthermore, because M1 and S2 share multiple common sources of excitatory input, defects in M1 could potentially be compensated by S2, or vice versa. The results reported here provide a starting point for investigating such synaptic plasticity at the cellular and subcellular levels.

Our subcellular analysis of the dendritic distributions of inputs from multiple sources extends prior observations using this technique (sCRACM) (Petreanu et al., 2009). More generally, this approach provides a way to investigate the hierarchical relationships between cortical areas at the crucial level of monosynaptic excitatory connectivity (Crick and Koch, 1998), a level that has recently become accessible using ChR2-based approaches in particular (Petreanu et al., 2007, 2009; Mao et al., 2011; Hooks et al., 2013; Yang et al., 2013). Although strong perisomatic responses were typical for all input pathways, each source displayed a distinct pattern of innervation distinguished by the way different subregions of the apical arbor were targeted. The differential targeting was particularly striking for corticocortical S2 $\rightarrow$ M1CSP versus thalamocortical VL $\rightarrow$ M1-CSP inputs, which showed an alternating pattern along the uppermost layers. Further work will be needed to test how pathway-specific targeting of different dendritic domains of CSPs engages active conductances underlying their integrative properties (Spruston, 2008; Larkum, 2013).

\section{References}

Alloway KD (2008) Information processing streams in rodent barrel cortex: the differential functions of barrel and septal circuits. Cereb Cortex 18: 979-989. CrossRef Medline

Anderson CT, Sheets PL, Kiritani T, Shepherd GMG (2010) Sublayerspecific microcircuits of corticospinal and corticostriatal neurons in motor cortex. Nat Neurosci 13:739-744. CrossRef Medline

Bachmann LC, Lindau NT, Felder P, Schwab ME (2014) Sprouting of brain stem-spinal tracts in response to unilateral motor cortex stroke in mice. J Neurosci 34:3378-3389. CrossRef Medline

Brecht M, Sakmann B (2002) Dynamic representation of whisker deflection by synaptic potentials in spiny stellate and pyramidal cells in the barrels and septa of layer 4 rat somatosensory cortex. J Physiol 543:49-70. CrossRef Medline

Burton H (1986) Second somatosensory cortex and related areas. In: Cerebral cortex: sensory-motor areas and aspects of cortical connectivity (Jones EG, Peters A, eds), pp 31-98. New York: Plenum.

Carvell GE, Simons DJ (1986) Somatotopic organization of the second somatosensory area (SII) in the cerebral cortex of the mouse. Somatosens Res 3:213-237. CrossRef Medline

Carvell GE, Simons DJ (1987) Thalamic and corticocortical connections of the second somatic sensory area of the mouse. J Comp Neurol 265:409_ 427. CrossRef Medline

Casale EJ, Light AR, Rustioni A (1988) Direct projection of the corticospinal tract to the superficial laminae of the spinal cord in the rat. J Comp Neurol 278:275-286. CrossRef Medline

Chakrabarti S, Alloway KD (2006) Differential origin of projections from SI barrel cortex to the whisker representations in SII and MI. J Comp Neurol 498:624-636. CrossRef Medline

Chen JL, Carta S, Soldado-Magraner J, Schneider BL, Helmchen F (2013) Behaviour-dependent recruitment of long-range projection neurons in somatosensory cortex. Nature 499:336-340. CrossRef Medline

Coogan TA, Burkhalter A (1993) Hierarchical organization of areas in rat visual cortex. J Neurosci 13:3749-3772. Medline

Crick F, Koch C (1998) Constraints on cortical and thalamic projections: the no-strong-loops hypothesis. Nature 391:245-250. CrossRef Medline

Donoghue JP, Parham C (1983) Afferent connections of the lateral agranular field of the rat motor cortex. J Comp Neurol 217:390-404. CrossRef Medline

Felleman DJ, Van Essen DC (1991) Distributed hierarchical processing in the primate cerebral cortex. Cereb Cortex 1:1-47. CrossRef Medline

Ghosh A, Sydekum E, Haiss F, Peduzzi S, Zörner B, Schneider R, Baltes C, Rudin M, Weber B, Schwab ME (2009) Functional and anatomical reorganization of the sensory-motor cortex after incomplete spinal cord injury in adult rats. J Neurosci 29:12210-12219. CrossRef Medline

Harrison TC, Ayling OG, Murphy TH (2012) Distinct cortical circuit mechanisms for complex forelimb movement and motor map topography. Neuron 74:397-409. CrossRef Medline

Herkenham M (1980) Laminar organization of thalamic projections to the rat neocortex. Science 207:532-535. CrossRef Medline

Hira R, Ohkubo F, Tanaka YR, Masamizu Y, Augustine GJ, Kasai H, Matsuzaki M (2013) In vivo optogenetic tracing of functional corticocortical connections between motor forelimb areas. Front Neural Circuits 7:55. CrossRef Medline

Hooks BM, Hires SA, Zhang YX, Huber D, Petreanu L, Svoboda K, Shepherd GMG (2011) Laminar analysis of excitatory local circuits in vibrissal motor and sensory cortical areas. PLoS Biol 9:e1000572. CrossRef Medline

Hooks BM, Mao T, Gutnisky DA, Yamawaki N, Svoboda K, Shepherd GMG (2013) Organization of cortical and thalamic input to pyramidal neurons in mouse motor cortex. J Neurosci 33:748-760. CrossRef Medline

Hunnicutt BJ, Long BR, Kusefoglu D, Gertz KJ, Zhong H, Mao T (2014) A comprehensive thalamocortical projection map at the mesoscopic level. Nat Neurosci 17:1276-1285. CrossRef Medline

Jeffery ND, Fitzgerald M (1999) Lack of topographical organisation of the corticospinal tract in the cervical spinal cord of the adult rat. Brain Res 833:315-318. CrossRef Medline

Jones EG (2007) The thalamus, Ed 2. Cambridge: Cambridge UP.

Kinnischtzke AK, Simons DJ, Fanselow EE (2014) Motor cortex broadly engages excitatory and inhibitory neurons in somatosensory barrel cortex. Cereb Cortex 24:2237-2248. CrossRef Medline

Larkum M (2013) A cellular mechanism for cortical associations: an orga- 
nizing principle for the cerebral cortex. Trends Neurosci 36:141-151. CrossRef Medline

Li CX, Waters RS (1991) Organization of the mouse motor cortex studied by retrograde tracing and intracortical microstimulation (ICMS) mapping. Can J Neurol Sci 18:28-38. Medline

Liang FY, Moret V, Wiesendanger M, Rouiller EM (1991) Corticomotoneuronal connections in the rat: evidence from double-labeling of motoneurons and corticospinal axon arborizations. J Comp Neurol 311:356-366. CrossRef Medline

Liao CC, Yen CT (2008) Functional connectivity of the secondary somatosensory cortex of the rat. Anat Rec (Hoboken) 291:960-973. CrossRef Medline

Li XG, Florence SL, Kaas JH (1990) Areal distributions of cortical neurons projecting to different levels of the caudal and spinal cord in rats. Somatosens Mot Res 7:315-335. CrossRef Medline

Mao T, Kusefoglu D, Hooks BM, Huber D, Petreanu L, Svoboda K (2011) Long-range neuronal circuits underlying the interaction between sensory and motor cortex. Neuron 72:111-123. CrossRef Medline

Neher RA, Mitkovski M, Kirchhoff F, Neher E, Theis FJ, Zeug A (2009) Blind source separation techniques for the decomposition of multiply labeled fluorescence images. Biophys J 96:3791-3800. CrossRef Medline

Nudo RJ, Masterton RB (1990) Descending pathways to the spinal cord: III. Sites of origin of the corticospinal tract. J Comp Neurol 296:559-583. CrossRef Medline

Paxinos G, Franklin KBJ (2001) The mouse brain in stereotaxic coordinates, Ed 2. London: Academic.

Petreanu L, Huber D, Sobczyk A, Svoboda K (2007) Channelrhodopsin-2assisted circuit mapping of long-range callosal projections. Nat Neurosci 10:663-668. CrossRef Medline

Petreanu L, Mao T, Sternson SM, Svoboda K (2009) The subcellular organization of neocortical excitatory connections. Nature 457:1142-1145. CrossRef Medline

Pierret T, Lavallée P, Deschênes M (2000) Parallel streams for the relay of vibrissal information through thalamic barreloids. J Neurosci 20:74557462. Medline

Pouchelon G, Gambino F, Bellone C, Telley L, Vitali I, Lüscher C, Holtmaat A, Jabaudon D (2014) Modality-specific thalamocortical inputs instruct the identity of postsynaptic L4 neurons. Nature 511:471-474. CrossRef Medline

Romo R, Salinas E (2001) Touch and go: decision-making mechanisms in somatosensation. Annu Rev Neurosci 24:107-137. CrossRef Medline

Saito T, Nakatsuji N (2001) Efficient gene transfer into the embryonic mouse brain using in vivo electroporation. Dev Biol 240:237-246. CrossRef Medline

Sato TR, Svoboda K (2010) The functional properties of barrel cortex neurons projecting to the primary motor cortex. J Neurosci 30:4256-4260. CrossRef Medline

Schindelin J, Arganda-Carreras I, Frise E, Kaynig V, Longair M, Pietzsch T, Preibisch S, Rueden C, Saalfeld S, Schmid B, Tinevez JY, White DJ, Hartenstein V, Eliceiri K, Tomancak P, Cardona A (2012) Fiji: an opensource platform for biological-image analysis. Nat Methods 9:676-682. CrossRef Medline

Schneider CA, Rasband WS, Eliceiri KW (2012) NIH Image to ImageJ: 25 years of image analysis. Nat Methods 9:671-675. CrossRef Medline

Shepherd GMG, Svoboda K (2005) Laminar and columnar organization of ascending excitatory projections to layer $2 / 3$ pyramidal neurons in rat barrel cortex. J Neurosci 25:5670-5679. CrossRef Medline
Shepherd GMG, Stepanyants A, Bureau I, Chklovskii D, Svoboda K (2005) Geometric and functional organization of cortical circuits. Nat Neurosci 8:782-790. CrossRef Medline

Smith JB, Alloway KD (2013) Rat whisker motor cortex is subdivided into sensory-input and motor-output areas. Front Neural Circuits 7:1-15. CrossRef Medline

Spruston N (2008) Pyramidal neurons: dendritic structure and synaptic integration. Nat Rev Neurosci 9:206-221. CrossRef Medline

Suter BA, O'Connor T, Iyer V, Petreanu LT, Hooks BM, Kiritani T, Svoboda K, Shepherd GMG (2010) Ephus: multipurpose data acquisition software for neuroscience experiments. Front Neurosci Methods 4:1-12. CrossRef Medline

Suter BA, Migliore M, Shepherd GMG (2013) Intrinsic electrophysiology of mouse corticospinal neurons: a class-specific triad of spike-related properties. Cereb Cortex 23:1965-1977. CrossRef Medline

Tabata H, Nakajima K (2001) Efficient in utero gene transfer system to the developing mouse brain using electroporation: visualization of neuronal migration in the developing cortex. Neuroscience 103:865-872. CrossRef Medline

Tabata H, Nakajima K (2008) Labeling embryonic mouse central nervous system cells by in utero electroporation. Dev Growth Differ 50:507-511. CrossRef Medline

Tennant KA, Adkins DL, Donlan NA, Asay AL, Thomas N, Kleim JA, Jones TA (2011) The organization of the forelimb representation of the C57BL/6 mouse motor cortex as defined by intracortical microstimulation and cytoarchitecture. Cereb Cortex 21:865-876. CrossRef Medline

Theyel BB, Llano DA, Sherman SM (2010) The corticothalamocortical circuit drives higher-order cortex in the mouse. Nat Neurosci 13:84-88. CrossRef Medline

Tseng GF, Prince DA (1993) Heterogeneity of rat corticospinal neurons. J Comp Neurol 335:92-108. CrossRef Medline

Ueta Y, Hirai Y, Otsuka T, Kawaguchi Y (2013) Direction- and distancedependent interareal connectivity of pyramidal cell subpopulations in the rat frontal cortex. Front Neural Circuits 7:164. CrossRef Medline

Ueta Y, Otsuka T, Morishima M, Ushimaru M, Kawaguchi Y (2014) Multiple layer 5 pyramidal cell subtypes relay cortical feedback from secondary to primary motor areas in rats. Cereb Cortex 24:2362-2376. CrossRef Medline

Weiler N, Wood L, Yu J, Solla SA, Shepherd GMG (2008) Top-down laminar organization of the excitatory network in motor cortex. Nat Neurosci 11:360-366. CrossRef Medline

Wood L, Gray NW, Zhou Z, Greenberg ME, Shepherd GMG (2009) Synaptic circuit abnormalities of motor-frontal layer $2 / 3$ pyramidal neurons in an RNA interference model of methyl-CpG-binding protein 2 deficiency. J Neurosci 29:12440-12448. CrossRef Medline

Yamashita T, Pala A, Pedrido L, Kremer Y, Welker E, Petersen CC (2013) Membrane potential dynamics of neocortical projection neurons driving target-specific signals. Neuron 80:1477-1490. CrossRef Medline

Yang W, Carrasquillo Y, Hooks BM, Nerbonne JM, Burkhalter A (2013) Distinct balance of excitation and inhibition in an interareal feedforward and feedback circuit of mouse visual cortex. J Neurosci 33:17373-17384. CrossRef Medline

Yu J, Anderson CT, Kiritani T, Sheets PL, Wokosin DL, Wood L, Shepherd GMG (2008) Local-circuit phenotypes of layer 5 neurons in motorfrontal cortex of YFP-H mice. Front Neural Circuits 2:1-8. CrossRef Medline 